\title{
Behind the scenes of spin-forbidden decay pathways in transition metal complexes
}

Moitra, Torsha; Karak, Pijush; Chakraborty, Sayantani; Ruud, Kenneth; Chakrabarti, Swapan

Published in:

Physical Chemistry Chemical Physics

Link to article, DOI:

$10.1039 / \mathrm{d} 0 \mathrm{cp} 05108 \mathrm{j}$

Publication date:

2021

Document Version

Peer reviewed version

Link back to DTU Orbit

Citation (APA):

Moitra, T., Karak, P., Chakraborty, S., Ruud, K., \& Chakrabarti, S. (2021). Behind the scenes of spin-forbidden decay pathways in transition metal complexes. Physical Chemistry Chemical Physics, 23, 59-81. https://doi.org/10.1039/d0cp05108j

\section{General rights}

Copyright and moral rights for the publications made accessible in the public portal are retained by the authors and/or other copyright owners and it is a condition of accessing publications that users recognise and abide by the legal requirements associated with these rights.

- Users may download and print one copy of any publication from the public portal for the purpose of private study or research.

- You may not further distribute the material or use it for any profit-making activity or commercial gain

- You may freely distribute the URL identifying the publication in the public portal 


\title{
Journal Name
}

\section{ARTICLE TYPE}

Cite this: DOI: $00.0000 / x x x x x x x x x x$

\section{Behind the scenes of spin-forbidden decay pathways in transition metal complexes}

\author{
Torsha Moitra, ${ }^{a}$ Pijush Karak, ${ }^{b}$, Sayantani Chakraborty, ${ }^{b}$ Kenneth Ruud ${ }^{* c}$ and Swapan \\ Chakrabarti*b
}

Received Date

Accepted Date

DOI: $00.0000 / x x x x x x x x x x$

\begin{abstract}
The interpretation of the ultrafast photophysics of transition metal complexes following photoabsorption is quite involved as the heavy metal center leads to a complicated and entangled singlet-triplet manifold. This opens up multiple pathways for deactivation, often with competitive rates. As a result, intersystem crossing (ISC) and phosphorescence are commonly observed in transition metal complexes. A detailed understanding of such excited-state structure and dynamics calls for state-of-the-art experimental and theoretical methodologies. In this review, we delve into the inability of non-relativistic quantum theory to describe spin-forbidden transitions, which can be overcome by taking into account spin-orbit coupling and whose importance grows with increasing atomic number. We present the quantum chemical theory of phosphorescence and ISC together with illustrative examples. Finally, a few applications are highlighted, bridging the gap between theoretical studies and experimental applications, such as photofunctional materials.
\end{abstract}

\section{Introduction}

The last decade can be unambiguously termed as the era of transition metal complexes. The advent of ultrafast spectroscopy has led to an improved understanding of the photophysics of transition metal complexes. The properties of transition metal complexes are fascinating not only because of their extensive use in opto-electronic materials, molecular electronics, biology, photochemistry and many more, but their photophysical properties also call for revisiting fundamental concepts.1.21

Early studies of transition metal complexes was primarily confined to and dominated by photosubstitution reactions typical of ligand field (LF) excited-state reactivities of metal-carbonyl complexes. $\stackrel{22 \mid 23}{ }$ In 1971, Adamson and coworkers ${ }^{24}$ opened up a totally new and fascinating area of research in luminescent transition metal complexes and their photochemistry by studying the photoredox reactivities attributed to the metal-to-ligand charge transfer (MLCT) excited state of ruthenium (II) bipyridine complexes. This was followed by the emergence of a new branch of supra-molecular photochemistry. 25,31 This rapidly growing field has been developed with the aid of sophisticated ligand-field theory and rigorous insight into electronic transitions and spectro-

\footnotetext{
a DTU Chemistry, Technical University of Denmark, Kemitorvet Bldg 207, DK-2800 Kongens Lyngby, Denmark.

${ }^{b}$ Department of Chemistry, University of Calcutta, 92 A. P. C. Road, Kolkata $\hat{a} \breve{A} S ̧$ 700009, West Bengal, India.

${ }^{c}$ Hylleraas Centre for Quantum Molecular Sciences, Department of Chemistry, Univer-

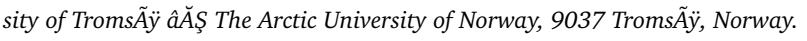

*Email: kenneth.ruud@uit.no, swcchem@caluniv.ac.in
}

scopic properties. $.25[32-39$ The field has also benefited significantly from the state-of-the-art discovery of high-precision spectroscopic techniques. 40 This ushered a demand for the advancement of computational tools for unravelling the microscopic origin of experimentally observed phenomena.

Electronically excited states formed by photoabsorption are rather short-lived and decay within a small period of time through a variety of deactivation processes. If the excited molecule goes back to its original initial ground-state electronic configuration, the phenomenon is a photophysical process. In contrast, when a molecule undergoes chemical changes, the corresponding dissipative pathway is termed as a photochemical process. Physical relaxation processes can be broadly categorised as being either intramolecular or intermolecular. In this context, intramolecular deactivation mechanisms can be accompanied with or without the emission of light, called radiative and non-radiative processes, respectively. In addition, intermolecular processes, as the name suggests, occur due to interactions between two or more molecules, leading to vibrational relaxations, energy transfer or electron transfer. All these processes are triggered by modifications of the electronic structure upon excitation and are accompanied by geometrical changes. A schematic representation of the intra-molecular photophysical events after excitation is shown by the modified Jablonski diagram ${ }^{41}$ in Fig. 1. named in honor of Aleksander Jablonski, who considered the metastable state as the origin of the delayed emission. This metastable state is now recognized as the triplet state. The electronic ground state of a molecule is typically of singlet character $\left(\mathrm{S}_{0}\right)$. Upon exposure 


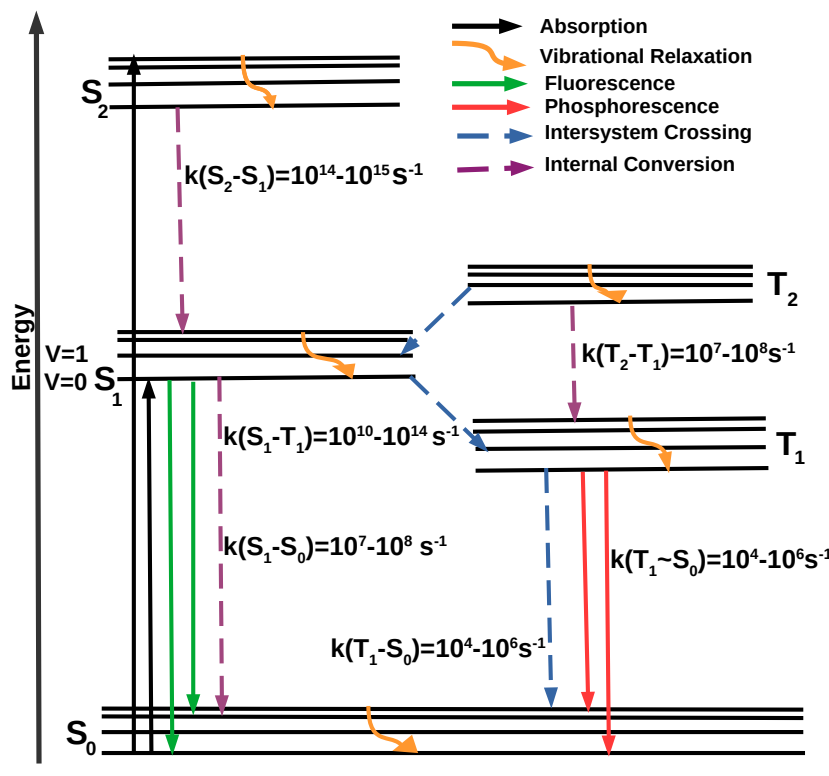

Fig. 1 Modified Jablonski diagram with rates typical of transition metal complexes.

to light, $\mathrm{S}_{0} \rightarrow \mathrm{S}_{n}$ absorption occurs, within a time-scale of a few femtoseconds. Nuclear changes are not feasible at such a short timescale and hence the molecule is usually excited to a higher vibrational level of the excited electronic state. The initially excited state has several possibilities for deactivation. It may undergo vibrational relaxation to the zero-point vibrational level of the $S_{n}$ state or a spin-forbidden intersystem crossing (ISC) to a near isoenergetic triplet excited state $\left(\mathrm{T}_{n}\right)$ leading to population growth within the triplet manifold. These singlet and triplet higher-energy excited states undergo non-radiative deactivation to the lowest energy excited state within its spin manifold $\left(S_{1}\right.$ or $\mathrm{T}_{1}$ ). This is the origin of Kasha's rule, which states that due to the rapid rate of deactivation to the lowest vibrational level of the $\mathrm{S}_{1}$ (or $\mathrm{T}_{1}$ ) states, luminescence emission will originate from the lowest vibrational level of $S_{1}$ and $T_{1}$. Emission from the $S_{1}$ and $\mathrm{T}_{1}$ states are referred to as fluorescence and phosphorescence, respectively. ${ }^{42}$ However, many instances of the breakdown of this rule has been documented. $43 \mid 48 / 48 / 49$

Due to spin conservation, transition between different spin states is spin-forbidden and thus phosphorescence is usually much less intense compared to the spin-allowed counterpart. Pioneering works by Terenin, Lewis and Kasha,, 50151 led to the understanding of the electronic origin of molecular phosphorescence.

The unique photophysical behavior of transition metal complexes is driven by the heavy atom-enhanced spin-orbit coupling (SOC) effect, which in a non-relativistic picture can be considered as leading to a mixing of different spin character into a spinpure (singlet or triplet) state. This leads to a fast rate of ISC and hence highly efficient population of the lowest-lying triplet state $\left(\mathrm{T}_{1}\right)$, followed by phosphorescence. This special characteristic has made these complexes promising candidates for a variety of applications. $52 \cdot 58$

Transition metal complexes have several advantages from the stand-point of designing smart optical display. Firstly, the phosphorescence from $\mathrm{d}^{6}, \mathrm{~d}^{8}$ or $\mathrm{d}^{10}$ electronic configurations usually leads to longer triplet-state lifetimes, allowing for their detection and offers more sensitivity towards time-resolved studies. Secondly, in contrast to their fluorescent organic counterparts, these complexes rarely suffer from self-quenching and homofluorescence resonance energy transfer. ${ }^{59}$ Thirdly, due to their ability to bind to a vast range of ligands, their photophysical characteristics can be fine-tuned more easily.

Heavy-metal complexes are a special class of compounds which has many different possibilities for excited-state character. These excited states include metal-to-ligand charge transfer (MLCT), ligand-to-metal charge transfer (LMCT), intra-ligand (IL), ligand-to-ligand charge transfer (LLCT), sigma-bond-toligand charge transfer (SBLCT), metal-metal-to-ligand charge transfer (MMLCT) and ligand-to-metal-metal charge transfer (LTMMCT). Notably, these characteristics can be readily perturbed by various metal-ligand combinations and by changing the environment of the complexes.

A clear acumen from theory and computation is necessary for rationalising the origin of experimental observations. Indeed, theoretical studies based on rate constants calculated using perturbation theory have played a decisive role. ${ }^{[60161}$ More recently, simulations combining quantum chemistry with nuclear dynamic studies have been developed. ${ }^{62}$ Considerable attention has been to the study of ISC processes using non-adiabatic molecular dynamics involving SOC. Also, ISC dynamics have been studied using wave-packet dynamics in the past couple of years.67,77

In this perspective, we present the theory of molecular spinforbidden transitions, namely, phosphorescence and ISC, which are the distinguishing characteristic of transition metal complexes. The concepts are further illustrated with specific examples demonstrating its wide-spread applicability.

\section{Spin-Orbit Coupling}

Spin-orbit coupling (SOC) is a relativistic effect emerging naturally from the full relativistic treatment of an electron,, 78 and couples the orbital angular momentum of an electron with its spin magnetic moment. In the case of atoms, the coupling strength scales with the fourth power of the nuclear charge, making it more significant for heavy transition metal atoms. Its magnitude typically varies from a few $\mathrm{meV}$ for elements of the 2 nd period to several $\mathrm{eV}$ for the valence shells of $6 \mathrm{p}$ elements. However, the nuclear charge is not the only parameter determining the SOC strengths, large SOC can also result when two electronic states of different spin multiplicities are close in energy.

The four-component electronic Dirac-Coulomb-Breit Hamiltonian is divided into a spin-free and a spin-dependent part. ${ }^{79 / 80}$ The latter accounts for the coupling of the electronic orbitals and spin degrees of freedom and leads to a mixing of states with different spin multiplicities. A fallacy of this separation approach is that the difference between the eigenfunctions of the electronic Hamiltonian based on multiplicity does not hold in the presence of spin-orbit coupling terms. However, for the sake of simplicity, we will in the following discussions assume that it is retained even when the SOC term is included. 
The SOC operator is most often expressed in terms of the BreitPauli (BP) operator,

$$
\begin{aligned}
\hat{H}_{\mathrm{SO}}^{\mathrm{BP}} & =\frac{1}{2 m_{e}^{2} c^{2}} \sum_{I} \sum_{i} \frac{Z_{I}}{r_{i I}^{3}}\left(\hat{\mathbf{r}}_{i I} \times \hat{\mathbf{p}}_{i}\right) \cdot \hat{\mathbf{s}}_{i} \\
& -\frac{1}{2 m_{e}^{2} c^{2}} \sum_{i} \sum_{j \neq i} \frac{1}{r_{i j}^{3}}\left(\hat{\mathbf{r}}_{i j} \times \hat{\mathbf{p}}_{i}\right) \cdot\left(\hat{\mathbf{s}}_{i}+2 \hat{\mathbf{s}}_{j}\right)
\end{aligned}
$$

where $m_{e}$ and $c$ are the electron mass and speed of light, respectively, and $Z$ is the nuclear charge. Capital and small letters are used to denote nuclei and electrons, respectively. $\hat{\mathbf{s}}_{i}$ is the spin operator of electron $i$. $\left(\hat{\mathbf{r}}_{i I} \times \hat{\mathbf{p}}_{i}\right)$ and $\left(\hat{\mathbf{r}}_{i j} \times \hat{\mathbf{p}}_{i}\right)$ are the angular momenta of electron $i$ with respect to nucleus $I$ and electron $j$, respectively.

The first term in Eq. 1 is the one-electron term describing the interaction of the spin magnetic moment of an electron with the magnetic moment induced by it while orbiting in the nuclear electrostatic field. This involves only spin-same-orbit coupling terms and arises from the electron-nuclear Coulomb attraction. The second term is the two-electron contribution and brings in contributions from both the spin-same-orbit and spin-other-orbit couplings. It relates to the interaction of the spin magnetic moment of the electron with the orbital magnetic moment of another electron. The spin-same-orbit part of the two-electron term originates from the transformation of the two-electron Coulomb interaction, whereas the spin-other-orbit term comes from the Gaunt interaction term. The Breit-Pauli spin-orbit operator has found widespread use, in particular in approaches based on perturbation or response theory. However, it is unbounded from below and can lead to a variational collapse in molecular calculations.

It is evident from Eq. 1 that the one-electron term has a stronger dependence on the nuclear charge, and surpasses the contribution from the two-electron term for high values of $Z$. In the case of $6 d$ elements like Pt, the two-electron term reduces the magnitude of the one-electron term by approximately $10 \%$. Thus, the second term can be neglected as an approximation to the BP operator for heavy elements. The two-electron term cannot, however, be neglected for lighter elements, where it leads to a significant screening of the one-electron part.

Various ways to approximate the one electron operator has been devised, $\frac{8182}{82}$ the most rigorous being the spin-orbit-meanfield (SOMF) Hamiltonian, designed by Hess et al. ${ }^{83}$ In this method, the two-electron spin-orbit interaction of partially filled orbitals is averaged for $\alpha$ and $\beta$ spin orientations before spin integration. This can be thought of as an extension of the frozen-core approximation to all-electron calculations. Benchmark calculations have shown that the accuracy of the SOMF operator corresponds closely to that of the full BP operator for heavy atoms. Several flavours of this approximate technique have been developed for use with internally contracted multireference configuration interaction (MRCI) and complete active space self-consistent field (CASSCF) wavefunctions. $\frac{84}{}$ A partial two-electron (p2E) operator has also been defined by Fedorov and Gordon. ${ }^{85}$ Recently, another mean-field approach for the two-electron contribution has been developed by Pokhilko et al. $\frac{86}{}$ and in this approach, the elegant use of Wigner-Eckart's theorem helps overcome the ex- plicit evaluation of all the multiplet components and the formalism is implemented within the equation of motion coupled cluster method. Apart from the computational efficiency, this approach also resolves the phase issue arising upon separation of nuclear and electronic degrees of freedom within Born-Oppenheimer approximation.

If we consider the effect of the two-electron spin-orbit term as a form of screening, an empirical approach for constructing an effective one-electron spin-orbit coupling operator is to emulate the two-electron contributions by an effective nuclear charge $\left(Z^{\text {eff }}\right)$

$$
\hat{H}_{\mathrm{SO}}^{\mathrm{eff}}=\frac{1}{2 m_{e}^{2} c^{2}} \sum_{I} \sum_{i} \frac{Z_{I, l}^{\mathrm{eff}}}{\hat{r}_{I i_{I}}^{3}} \hat{l}_{I i_{I}} \hat{s}_{i_{I}}
$$

Here $i_{I}$ denotes an electron occupying an orbital located at center $I$. Blume, Watson and Peierls showed that Eq. 2 only partially accounts for the two-electron contribution. $\frac{87}{\text { The use of }}$ this operator is limited by it's dependence on the effective nuclear charge from a particular state, which varies considerably for $d^{n}$ configurations. ${ }^{88}$ Despite these fundamental deficiencies, $\hat{H}_{\mathrm{SO}}^{\text {eff }}$ has been used extensively for studies of transition metal complexes by appropriate parameterization of $Z^{\text {eff }}$ to fit experimental fine-structure splittings of one or more electronic states of an atom. 89

The concept of an effective spin-orbit coupling operator has been combined with the concept of effective core potential (ECP). Here, $Z^{\text {eff }}$ has no physical meaning and is a purely empirical parameter. 9293 In the vicinity of a nuclear center, the amplitudes of pseudo-orbitals are small and the effective charge becomes very large. Most commonly, in the spin-orbit pseudo-potential approach, the $\frac{1}{r^{3}}$ dependence is dropped and the SOC operator is shifted to the valence region. Teichteil et al. has fit the spin-orbit pseudo-operator such that its action on a pseudo-orbital appropriately reproduces the effect of the true spin-orbit operator of the corresponding all-electron orbital.94

An alternative approach of including effects of inner shells on the valence properties is to use the $a b$ initio model potential (AIMP) method. ${ }^{95}$ Contrary to the pseudo-orbitals, the AIMP orbitals retain the complete nodal structure of the all-electron operators. A combination of SOMF Hamiltonian alongside ECP has also been used. 96

SOC matrix elements are available for both quantum chemical methods and semi-empirical procedures. There are two main routes to calculate SOC matrix elements. One is to treat electron correlation and SOC simultaneously in a single step and second, to evaluate the SOC in a basis of correlated molecular wavefunctions (two-step procedure). The utility of the two approaches depends on the system under study and the property to be determined. Variational perturbation theory, response theory and multi-reference spin-orbit configuration interaction (MRSOCI) are some of the one-step methods. ${ }^{97}$ These are beneficial for calculating second-order spin-dependent properties requiring an infinite summation over zero-order states within the RayleighSchrödinger perturbation theory. MRSOCI wavefunction for the calculation of phosphorescence is especially beneficial. 98 Timedependent Density functional theory (TD-DFT) coefficients are 
also used to calculate SOC matrix elements between singlet- and triplet-excited states. ${ }^{99}$ Two-step protocols offer the possibility of computing the diagonal matrix elements corresponding to spinfree excitation energies and the SOC matrix elements at different levels of theory. $100 \mid 101$

A popular method for computing relativistic effects of heavy-metal systems is the zeroth-order regular approximation (ZORA).102 105 The method transforms the four-component Dirac Hamiltonian to a two-component form, by replacing the expansion of $(E-V) / 2 c^{2}$ term by $E /\left(2 c^{2}-V\right)$ for a relativistic particle moving in a Coulomb potential. This variationally stable and self-consistent ZORA method has further been simplified by Wang and Ziegler, 106 where the lowest single-group excited states are evaluated at the scalar relativistic, time-dependent density functional theory(sr-TDDFT) level and spin-orbit coupling (SOC) is then included as a perturbation using the reference orbitals, obtained in a sr-TDDFT computation. The beauty of the method lies in the fact that the composition of the double-group excited states can automatically be evaluated from the single-group singlet and triplet excited states even though the relativistic wave functions are characterized by half-integral angular momentum quantum number and, in principle, it should have a point double-group symmetry.

The SOC operator must also be transformed according to the ZORA approximation and then becomes

$$
\hat{H}_{S O}=i \sigma\left(\mathbf{p} \times \frac{c^{2}}{2 c^{2}-V} \mathbf{p}\right)
$$

where $\sigma$ and $\mathbf{p}$ are the Pauli spin matrix vectors and moment operator, respectively. The computational cost of this perturbativeSOC (p-SOC) approach is significantly lower than self-consistent SOC-TDDFT method and has a negligible effect on the quality of the calculated results. 107 As a consequence, p-SOC method is now widely used to compute the SOC matrix elements of large transition metal complexes. $108-110$ However, this approach should in principle be always used in combination with specially designed ZORA basis, as one might otherwise get significant errors.

There has as of yet not been any systematic studies of phosphorescence at the four-component relativistic level of theory, despite this being computationally more straightforward than when non-relativistic reference wave functions are used. In fourcomponent relativistic theory, transition moments and lifetimes are governed directly by dipole transition matrix elements, as the electronic states are no longer pure spin states. In a similar manner, ISC are governed by transition dipole moments between excited states. The reasons for the lack of use of four-component relativistic methods in the study of transition metal complexes. are three-fold: 1) relativistic four-component calculations of molecular properties have a much shorter history than non-relativistic molecular property calculations; 2) four-component relativistic calculations have until recently been significantly more computationally expensive than non-relativistic calculations; and 3) the noncollinear exchange-correlation kernels that have to be used because of the spin-orbit coupling. 111
However, developments suggests that this picture is changing, suggesting that four-component and approximate twocomponent relativistic calculations, in which spin-orbit effects are included variationally, may become much more commonplace in future. The first four-component relativistic timedependent density-functional theory (TDDFT) implementation was presented by Gao et al.112 113, but limited to excitation energies only. Bast, Jensen and Saue later reported a noncollinear implementation of TDDFT in which also transition moments could be obtained. 114 However, all these papers reported results for atoms and fairly small molecular systems. Very recently, an implementation of four-component relativistic TDDFT for both openand closed-shell systems was presented by Komorovsky, Cherry and Repisky. $\frac{115}{15}$ Although larger systems was studied in this article, the implementation was restricted to excitation energies only.

An alternative approach to address both excitation energies and transition moments is the complex polarization propagator. At the four-component level of theory, the first implementation was presented by Villaume, Saue and Norman 116 and applied to, for instance, the phosphorescence of transitional metal porphyrins. ${ }^{117}$ More recently a similar approach was implementd by Konecny et al.118 and employed on some transition metal complexes $\left(\left[\mathrm{M}(\mathrm{phen})_{3}\right]^{3+}(\mathrm{M}=\mathrm{Fe}, \mathrm{Ru}\right.$, Os $)$. These developments suggests that four-component relativistic calculations of phosphorescence and ISC may soon be within reach also for transition metal complexes.

SOC constants for $5 \mathrm{~d}$ elements are usually of the order of a few thousands $\mathrm{cm}^{-1} 119$ One might naively assume that SOC is large in all transition metal complexes, but this is not the case. The singlet to triplet transition under study must have a large metal contribution in order to have a substantial amount of SOC. 109$] 120$ For instance, the $\left\langle S_{1}\left|\hat{H}_{S O}\right| T_{1}\right\rangle$ and $\left\langle S_{1}\left|\hat{H}_{S O}\right| T_{2}\right\rangle$ values for (acetylacetonato)-bis(1-methyl-2-phenylimidazol)iridium(III) (aka N966) complex is 93 and $513 \mathrm{~cm}^{-1}$, respectively. 108 This stark difference is reasonable as only the latter matrix element involves charge transfer from the $5 \mathrm{~d}$ orbital of iridium to the acetylacetonato group. This different characteristics of the states are often used to preferentially select one transition pathway over another.

\section{Phosphorescence}

As per the IUPAC definition, phosphorescence can be defined phenomenologically as long-lived luminescence. According to mechanistic photochemistry, the term designates luminescence involving change in spin multiplicity, typically from a triplet to a singlet state, or vice versa. 121

Historically, the term has been used somewhat uncritically, and was initially used for all long-lived emission processes. The phenomenological definition as a distinction of the time duration of afterglow compared with fluorescence was used until recently and was a source of ambiguity. $\frac{122 \mid 123}{12}$ The term has been used several times to describe luminescence phenomena arising due to completely different photophysical mechanisms. ${ }^{124}$ Here, we concentrate on the well established mechanistic definition of phosphorescence and thereby only look at radiative transitions between different spin states. 
Theoretical understanding of the phenomenon requires analysis of the characteristics of the excited-state electronic structure. Typically, phosphorescence occurs from the lowest triplet state $\left(\mathrm{T}_{1}\right)$ as a consequence of IC within the triplet manifold. The three triplet sublevels are degenerate within non-relativistic quantum theory, whereas the degeneracy is lifted in the relativistic theory due to zero-field splitting effects.

In the following sections we describe the theoretical methodologies developed for the calculation of phosphorescence within the framework of non-relativistic wavefunction and densityfunctional theories, focusing on perturbation theory and the quadratic response theory formalism. Finally, we discuss the basis for estimating phosphorescence lifetimes as well as transition moments and intensities.

\subsection{Perturbation theory approach}

SOC leads to a mixing of the pure singlet $\left(S_{n}\right)$ and triplet $\left(T_{n}\right)$ states by some contributions from the other spin state. In the limit when the contribution of the singlet state towards the triplet state (and vice-versa) is small, the situation can be described by first-order perturbation theory. $\frac{125}{127}$ The total wave function of the triplet state $\left(\tilde{T}_{1}\right)$ can then be written as,

$$
\left|\tilde{T}_{1}\right\rangle=\left|T_{1}\right\rangle+\sum_{n} \frac{\left\langle S_{n}\left|\hat{H}_{S O}\right| T_{1}\right\rangle}{E\left(T_{1}\right)-E\left(S_{n}\right)}\left|S_{n}\right\rangle
$$

Similarly, the ground state is also perturbed by SOC and has some contributions from the triplet manifold.

$$
\left|\tilde{S}_{0}\right\rangle=\left|S_{0}\right\rangle+\sum_{n} \frac{\left\langle T_{n}\left|\hat{H}_{S O}\right| S_{0}\right\rangle}{E\left(S_{0}\right)-E\left(T_{n}\right)}\left|T_{n}\right\rangle
$$

Smaller singlet-triplet energy separation, and higher SOC between the opposite spin states, increases the mixed nature of the states. In the case of phosphorescence from the lowest energy triplet state $\left(\tilde{T_{1}} \rightarrow \tilde{S_{0}}\right)$, the rate of population decay is given by the electric transition dipole moment between the two states.

$$
\begin{aligned}
\left\langle\tilde{S}_{0}|\hat{\mu}| \tilde{T}_{1}\right\rangle & =\left(\left\langle T_{1}|\hat{\mu}| T_{1}\right\rangle-\left\langle S_{0}|\hat{\mu}| S_{0}\right\rangle\right) \frac{\left\langle T_{1}\left|\hat{H}_{S O}\right| S_{0}\right\rangle}{E\left(T_{1}\right)-E\left(S_{0}\right)} \\
& +\sum_{n} \frac{\left\langle S_{n}|\hat{\mu}| S_{0}\right\rangle\left\langle S_{n}\left|\hat{H}_{S O}\right| T_{1}\right\rangle}{E\left(T_{1}\right)-E\left(S_{n}\right)}+\sum_{n} \frac{\left\langle T_{1}|\hat{\mu}| T_{n}\right\rangle\left\langle T_{n}\left|\hat{H}_{S O}\right| S_{0}\right\rangle}{E\left(S_{0}\right)-E\left(T_{n}\right)}
\end{aligned}
$$

Recalling that the SOC matrix elements are imaginary, see Eq. 1 . one must also take into account the complex conjugate. The absolute value of the square of the transition moment is the same for both absorption and emission at a given geometry. The energy gap appearing in the denominator of Eq. 6 does not depend on the spin sub-levels as zero-field splitting is much smaller than the singlet-triplet energy gap.

The first term in Eq. 6 is a permanent dipole difference term of the pure spin states, while the second and third terms are guided by the spin-allowed transition moments. The dipole difference term plays a dominant role for heavy-metal complexes where the triplet state often has charge-transfer character. Metal-to-ligand charge-transfer states involve excitations from the heavy-atom center, leading to stronger SOC and thereby enhanced phospho- rescence quantum yield. $\frac{15[109] 128}{10}$ The second term in Eq. 6 is important for purely organic molecules.

It is worth noting that the triplet state is comprised of three sub-levels, each having a distinct decay rate with different polarization directions along the three Cartesian coordinate axes. Even though this is true for highly symmetric molecules, it is not so for systems with lower symmetry. For the latter, the quantization axes of the zero-field triplet sub-levels may not be the same as the symmetry axes, leading to emissions of mixed polarization.

\subsection{Response theory approach}

Response theory formalism ${ }^{129}$ is an alternative way of formulating time-dependent perturbation theory discussed that allows exact- and approximate-state theories to be formulated in the same way, differing mainly in the explicit parameterization of these states. Specific molecular properties are obtained as response functions, identified in an order expansion with respect to time or frequency of an observable quantity in terms of an applied perturbation. The external time-dependent perturbing field $\left(\hat{V}^{t}\right)$ generates a response on a molecular property $(\hat{\Omega})$, which can be defined as the time-dependent expectation value of an observable, such as the electric dipole moment.

$$
\begin{aligned}
\langle\psi(t)|\hat{\Omega}| \psi(t)\rangle & =\left\langle\psi^{(0)}|\hat{\Omega}| \psi^{(0)}\right\rangle \\
& +\left\langle\psi^{(1)}|\hat{\Omega}| \psi^{(0)}\right\rangle+\left\langle\psi^{(0)}|\hat{\Omega}| \psi^{(1)}\right\rangle \\
& +\left\langle\psi^{(2)}|\hat{\Omega}| \psi^{(0)}\right\rangle+\left\langle\psi^{(1)}|\hat{\Omega}| \psi^{(1)}\right\rangle+\left\langle\psi^{(0)}|\hat{\Omega}| \psi^{(2)}\right\rangle \\
& +\ldots
\end{aligned}
$$

were $\psi^{(n)}$ is the $\mathrm{n}$-th order perturbed wavefunction. This can be written in terms of response functions as,

$$
\begin{aligned}
\langle\psi(t)|\hat{\Omega}| \psi(t)\rangle & =\langle 0|\hat{\Omega}| 0\rangle+\int_{-\infty}^{\infty}\left\langle\left\langle\hat{\Omega} ; \hat{V}^{\omega_{1}}\right\rangle\right\rangle e^{-i \omega_{1}} d \omega_{1} \\
& +\frac{1}{2} \int_{-\infty}^{\infty} \int_{-\infty}^{\infty}\left\langle\left\langle\hat{\Omega} ; \hat{V}^{\omega_{1}} \hat{V}^{\omega_{2}}\right\rangle\right\rangle e^{-i\left(\omega_{1}+\omega_{2}\right)} d \omega_{1} d \omega_{2} \\
& +\frac{1}{6} \int_{-\infty}^{\infty} \int_{-\infty}^{\infty} \int_{-\infty}^{\infty}\left\langle\left\langle\hat{\Omega} ; \hat{V}^{\omega_{1}} \hat{V}^{\omega_{2}} \hat{V}^{\omega_{3}}\right\rangle\right\rangle \\
& e^{-i\left(\omega_{1}+\omega_{2}+\omega_{3}\right)} d \omega_{1} d \omega_{2} d \omega_{3}
\end{aligned}
$$

$\left\langle\left\langle\hat{\Omega} ; \hat{V}^{\omega_{1}}\right\rangle\right\rangle, \quad\left\langle\left\langle\hat{\Omega} ; \hat{V}^{\omega_{1}} \hat{V}^{\omega_{2}}\right\rangle\right\rangle$ and $\left\langle\left\langle\hat{\Omega} ; \hat{V}^{\omega_{1}} \hat{V}^{\omega_{2}} \hat{V}^{\omega_{3}}\right\rangle\right\rangle$ are the linear, quadratic and cubic response functions, respectively. These corresponds to changes induced on the observable property $\hat{\Omega}$, upon second-, third- or fourth-order perturbations of light-matter interactions.

For the study of phosphorescence, the SOC operator acts as a perturbation to the electric dipole operator $\left(\mu^{a}\right)$ and hence the observable to be computed is associated with the residue of the quadratic response function:

$$
\lim _{\omega \rightarrow \omega_{f}}\left(\omega-\omega_{f}\right)\left\langle\left\langle\hat{\mu}^{a} ; \hat{H}_{S O}, \hat{V}^{\omega_{2}}\right\rangle\right\rangle_{0, \omega}
$$


The response function (Eq. 9) corresponding to the transition moment is given by (using the Einstein summation convention for repeated indices),

$$
\begin{aligned}
\lim _{\omega \rightarrow \omega_{1}} & \left(\omega-\omega_{1}\right)\left\langle\left\langle\hat{\mu}^{a} ; \hat{H}_{S O}, \hat{V}^{\omega_{2}}\right\rangle\right\rangle_{0, \omega} \\
& =-N_{j}^{r}\left(\omega_{f}\right) H_{S O}^{[2]} X_{a f}-N_{j}^{S O}\left(\mathbf{r}_{j a}^{[2]}+\mathbf{r}_{a j}^{[2]}\right) X_{a f} \\
& +N_{j}^{r}\left(\omega_{f}\right)\left(E_{j m a}^{[3]}+E_{j a m}^{[3]}-\omega_{f} S_{j a m}^{[3]}\right) N_{m}^{S O} X_{a f}
\end{aligned}
$$

were the linear response vectors $N$ comes from solutions of the linear response equations:

$$
N^{r}\left(\omega_{f}\right)=\left[\left(E^{[2]}-\omega_{f} S^{[2]}\right)^{-1} r^{[1]}\right]^{*} \text { and } N^{S O}=\left(E^{[2]}\right)^{-1} H_{S O}^{[1]}
$$

$E^{[2]}$ and $S^{[2]}$ arises from the second-order variation with respect to wave function parameters of the Hamiltionan and overlap matrices, respectively. Solving the triplet excitation eigenvalue problem gives the triplet excitation vectors $\left(X_{f}\right)$ and frequencies $\left(\omega_{f}\right)$.

$$
\left(E^{[2]}-\omega_{f} S^{[2]}\right) X_{f}=0
$$

We do not elaborate further here on the mathematical complexities of Eqs. 10 and 11 and refer to Ref. 129 for more details. Instead, we would highlight the advantage of quadratic response theory for calculations of phosphorescence. A key element of response theory is that the usual sum-over-excited-state algorithm is replaced by a set of linear equations that can be performed without prior knowledge of the excited states. 130 This renders the method useful for treating large molecular systems described by approximate wave functions, and molecular properties can calculated analytically. $\frac{108}{131} 133$ We note that several other strategies are also available for the computation of phosphorescence phenomenon, such as the variational perturbation theory 134 and spin-orbit coupling configuration interaction (SOC-CI) ${ }^{135 \mid 136}$ approaches. Some of these become particularly important for systems with nearly degenerate electronic ground states and where the spin-orbit contributions arise largely from within this manifold of nearly degenerate electronic states.

\subsection{Lifetime}

The phosphorescence lifetime $\tau_{k}$ from one of the three sublevels $(k)$ of the lowest triplet state $\left(\mathrm{T}_{1}\right)$ is given by the inverse of the rate of spontaneous emission $\left(A_{k \rightarrow 0}\right)$.

$$
\frac{1}{\tau_{k}}=A_{k \rightarrow 0}=\frac{1}{3 \pi \varepsilon_{0} \hbar}\left(\frac{\omega}{c}\right)^{3} \sum_{a=x, y, z}\left|M_{a}^{k}\right|^{2}
$$

$M_{\alpha}^{k}$ is the electric dipole transition moment between the ground state and the triplet state. The lifetime has a cubic dependence on the radiation frequency $\omega$. The radiative lifetime $\tau$ of the triplet state in the high temperature limit is obtained by averaging over the three sublevels, assuming them to be equally populated due to the very small energy differences between the three spin sublevels and the available energy at temperatures normally used in experimental studies.

$$
\frac{1}{\tau_{a v}}=\frac{1}{3} \sum_{k=1}^{3} \frac{1}{\tau_{k}}
$$

For transition metal complexes, Eq. 14 is not strictly valid due to the potentially large zero-field splittings. This can be rectified by Boltzmann-weighted averaging over the individual spin sub-levels of the triplet state.

The lifetime of the triplet state is usually a few orders of magnitude larger than that of the singlet states, enabling a more detailed time-resolved study exploiting the triplet characteristics of the molecule. This has been extensively used in designing stateof-the-art molecular materials. $125[137 / 138$

\subsection{Intensity}

Due to the fact that spin is a good quantum number in nonrelativistic theory, whereas the spin-orbit interaction leads to an inherent mixing of states of different spin multiplicity in variationally optimized relativistic calculations, the calculation of phosphorescence transition moments and intensities differs between the relativistic and non-relativistic realms. Within the former, there is no difference between fluorescence and phosphorescence and the electric transition dipole transition moments are obtained as residues of the linear response function.

$$
\lim _{\omega \rightarrow \omega_{f 0}}\left(\omega_{f 0}-\omega\right) \alpha_{a b}(-\omega ; \omega)=\left\langle 0\left|\hat{\mu}_{a}\right| f\right\rangle\left\langle f\left|\hat{\mu}_{b}\right| 0\right\rangle
$$

$\alpha$ is here the electric dipole polarizability. On contrast, in the non-relativistic realm, invoking a perturbation treatment is necessary even when zero-field splitting is ignored. The first non-zero contribution to the transition moment comes from the first-order corrected wave functions. The sublevels of the triplet state are energetically degenerate, hence the final expression becomes,

$$
\begin{aligned}
M_{a}^{k} & =\sum_{n=0}^{\infty} \frac{\left\langle S_{0}\left|\hat{\mu}_{a}\right| S_{n}\right\rangle\left\langle S_{n}\left|\hat{H}_{S O}\right| T_{1}^{k}\right\rangle}{E\left(S_{n}\right)-E\left(T_{1}\right)} \\
& +\sum_{n=1}^{\infty} \frac{\left\langle S_{0}\left|\hat{H}_{S O}\right| T_{n}\right\rangle\left\langle T_{n}\left|\hat{\mu}_{a}\right| T_{1}^{k}\right\rangle}{E\left(T_{n}\right)-E\left(S_{0}\right)}
\end{aligned}
$$

The summation over the intermediate triplet states runs over all the three sub-levels of the triplet state. The oscillator strength is thus given by,

$$
f_{T \rightarrow 0}=\frac{2}{3}\left(E_{T}-E_{0}\right) \sum_{k=1}^{3} \sum_{a=x, y, z}\left(M_{a}^{k}\right)^{2}
$$

$M_{a}^{k}$ can be identified from the single residue of a quadratic response function, so the final expression reads

$$
M_{a}^{k}=\lim _{\omega \rightarrow \omega_{f 0}} \frac{\hbar\left(\omega_{f 0}-\omega\right)\left\langle\left\langle\hat{\mu}_{a} ; \hat{H}_{S O}^{k}, \hat{\Omega}\right\rangle\right\rangle_{0, \omega}}{\langle f|\hat{\Omega}| 0\rangle}
$$

\subsection{Some examples}

The success of prevalent phosphorescence from transition metal complexes is due to the comparable rate constants for radiative 

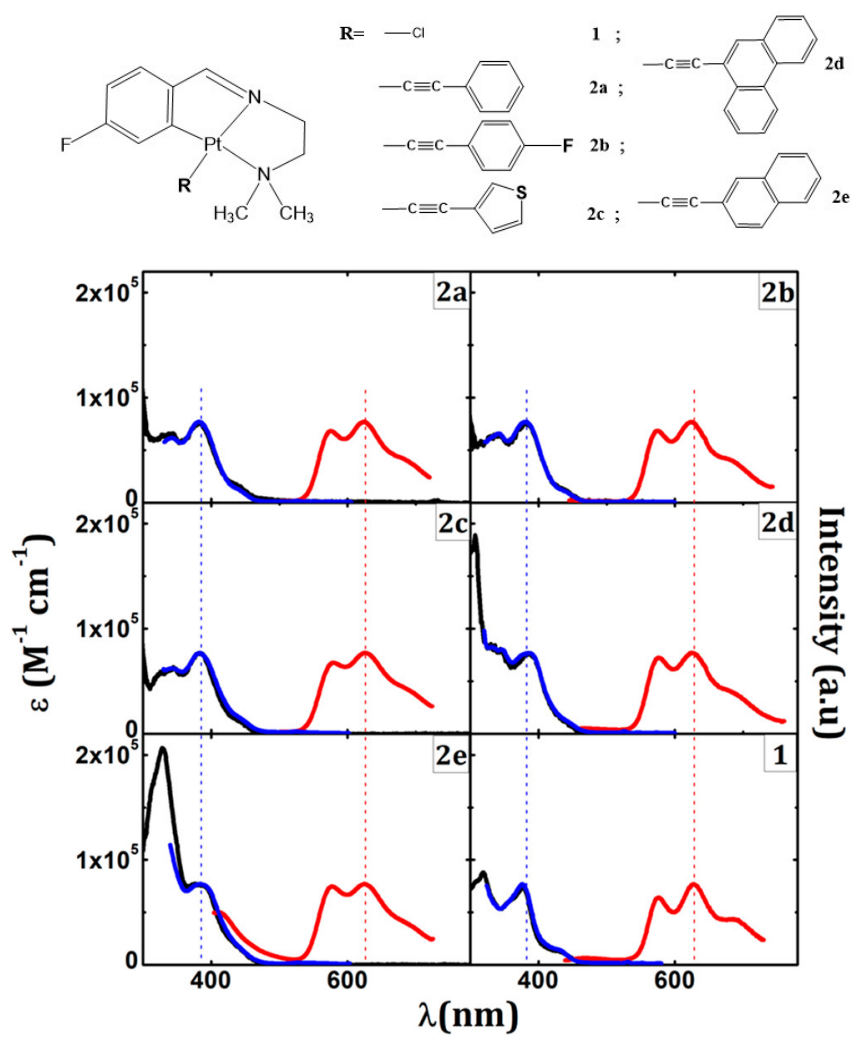

Fig. 2 Chemical structures of alkynly cylocmetalated $\mathrm{Pt}(\mathrm{II})$ complexes (top). Absorption (black) with phosphorescence excitation (blue) and phosphorescence (red) spectra are shown at the bottom. Adapted with permission from Ref. 139 Copyright 2020 American Chemical Society.

and non-radiative transitions from $\mathrm{T}_{1}$ state. From Hund's rule, it follows that the triplet sub-levels are lower in energy than the first singlet state, and phosphorescence therefore occurs at lower energies in comparison to fluorescence. Also, in principle, phosphorescent molecules can achieve 100\% [75\% $\mathrm{T}_{1} \rightarrow \mathrm{S}_{0}+25 \%$ $\mathrm{S}_{1} \rightarrow \mathrm{S}_{0}$ ] quantum efficiency.

Extensive theoretical studies have been performed on Ir(III) complexes with organic ligands, and we refer to Ref. 142 for more details. We instead focus our discussion on Pt complexes, as their phosphorescence properties can be fine-tuned to meet a variety of different requirements. Also, the thermal stability and charge neutrality of cyclometallated Pt(II) complexes render them good candidates as triplet emitters. $\frac{143144}{14}$ The triplet state population rates of Pt(II) complexes are moderate and thus structure or environment alterations can swing the balance towards purely phosphorescent or dual emissive luminescence behavior.

Purely phosphorescent alkynyl cyclometalated Pt complexes (Fig. 2) have recently been investigated by Lázaro et al. 139 These complexes exhibit a phosphorescence maximum at $625 \mathrm{~nm}$ with several vibrational fine structures, corresponding to $\mathrm{C}-\mathrm{C}$ and $\mathrm{C}$ $\mathrm{N}$ stretching modes. DFT and TDDFT calculations show that the transition is mediated by IL states mixed with MLCT character. The phosphorescence quantum efficiency and triplet lifetimes can be further enhanced by making the complex more rigid by ligating with aromatic ligands. 145
On the other hand, BODIPY and thioxanthonyl platinum(II) complexes show dual emissive behavior with fluorescence at 450$490 \mathrm{~nm}$ and phosphorescence at 510-650 nm, as shown in Fig. $3140[141$ The influence of the ligand is noticeable for the BODIPY ligand complex. The cis isomers have very low phosphorescence intensity in comparison to their trans counterparts. The calculations by Geist et al. 140 shows that frontier molecular orbitals involved, have the majority of the spin density on the BODIPY moiety with only little contribution from the $d \pi$ orbital of Pt. This spin density distribution is prototypical scenario favouring dual emission.

The cases discussed so far may trick one to think that phosphorescent wavelengths of transition metal complexes lie in the red and near infra-red region of the optical spectra. However, more recently a variety of blue phosphorescent Pt (II) complexes have been designed. 146 Bidentate or tridentate Pt(II) complexes have low quantum efficiency and poor stabilities, and more rigid tetradentate complexes are therefore the usual choice. The tetradentate Pt(II) complexes are mainly of two types, metallochelates where the chelating ligands are linked by $\pi$-conjugation, and macrocylic complexes. The second class of complexes are very rigid and thus ensure high quantum efficiency and stability. $146-152$

In order to demonstrate the generation of different visible light phosphorescent complexes, based purely on design, we use a series of tetradentate Pt(II) emitters having fused 5/6/6 metallocycles (Fig. 4). 146 The photoluminescence spectra of these complexes at $77 \mathrm{~K}$ and room temperature are shown in Fig. 5. In comparison to $\mathrm{Pt}(2-\mathrm{ptz})$ complexes, these complexes display red shifts to varying degrees. Photoluminescence spectra at $77 \mathrm{~K}$ shows a structured profile with a dominant peak followed by two or five fine-structure bandss. However, at room temperature, a broad Gaussian type emission is seen. The strong rigidochromic shift of 8-52 $\mathrm{nm}$ exhibited by these molecules are a hallmark of significant ${ }^{1}$ MLCT $/{ }^{3}$ MLCT characters in the lowest excited states.

\section{Intersystem Crossing}

Intersystem crossing (ISC) is the near isoenergetic radiationless transition between two electronic states having different spin multiplicities. Even though it is described as a spin-forbidden singlet-triplet transition, like phosphorescence, the physical origin of the two is different. In particular, phosphorescence is guided by the transition dipole moment between the SOC perturbed singlet and triplet states, while ISC is fueled by the strength of the spin-orbit interaction between the initial and the final electronic states. ISC plays a key role in driving phosphorescence as it transfers population from the initially excited singlet state to the triplet manifold. Hence, we present here a detailed account on the developments of theoretical protocols for the calculation of the rate of ISC.

The study of the rate of population transfer between the initial and final states involved in an electronic transition can be studied by two approaches, nuclear dynamics based methods and perturbation theory-based approaches. The former involves considering the explicit nuclear motion over the potential energy surface, thereby following the nuclear dynamics of the molecule. This 

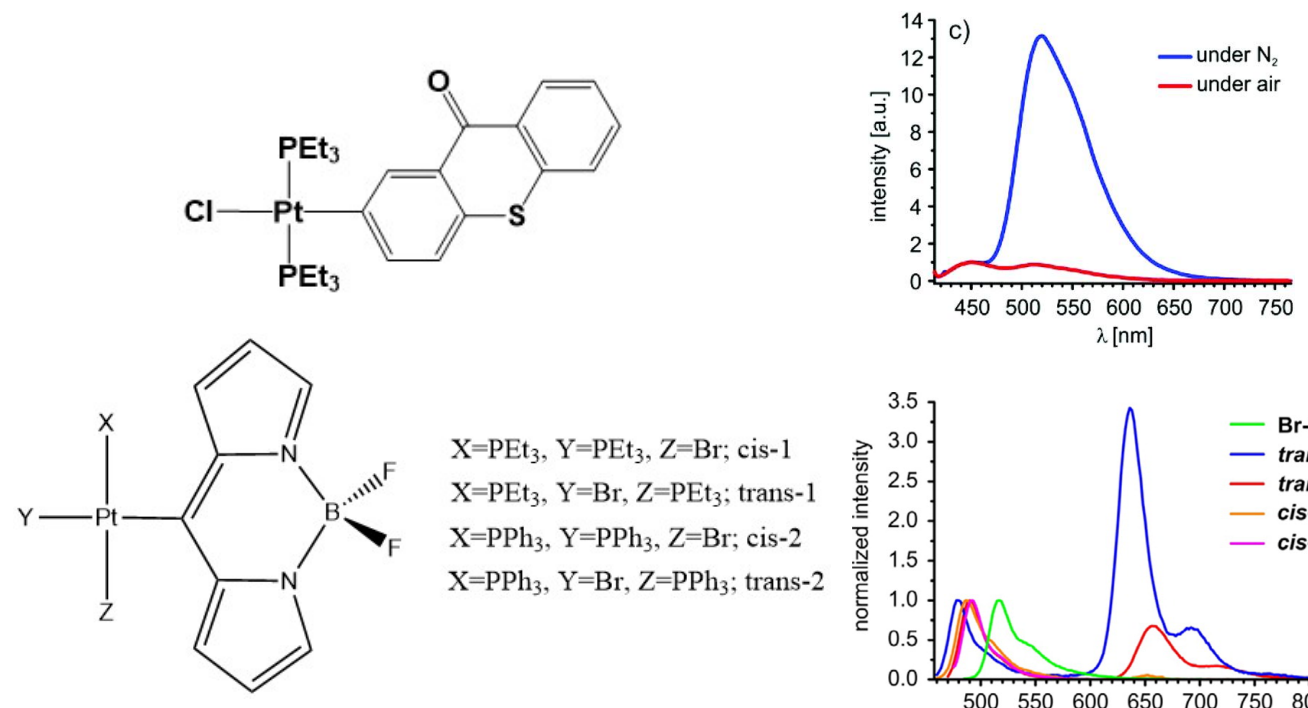

$\mathrm{X}=\mathrm{PEt}_{3}, \mathrm{Y}=\mathrm{PEt}_{3}, \mathrm{Z}=\mathrm{Br}$; cis-1

$\mathrm{X}=\mathrm{PEt}_{3}, \mathrm{Y}=\mathrm{Br}, \mathrm{Z}=\mathrm{PEt}_{3}$; trans-1

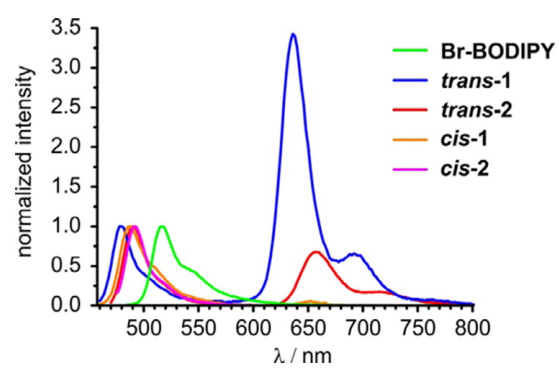

Fig. 3 Chemical structure and photoluminescence spectra of Pt thioxanthonyl (top) and BODIPPY (bottom) complexes. Adapted with copyright from Ref. 140 and 141 Copyright 2015 American Chemical Society and 2015 Royal Society of Chemistry.

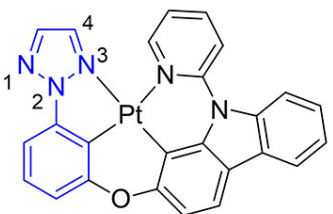

$\mathrm{Pt}(2-p t z-\mathrm{O}-\mathrm{CbPy})$

$\operatorname{Pt}(2-p t z)\left(32.1^{\circ}\right)$

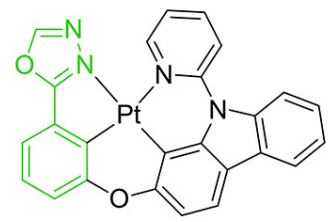

$\mathrm{Pt}($ podz-O-CbPy)

$\operatorname{Pt}($ podz $)\left(17.3^{\circ}\right)$

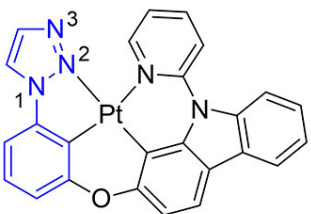

$\mathrm{Pt}(1-\mathrm{ptz}-\mathrm{O}-\mathrm{CbPy})$

$\operatorname{Pt}(1-p t z)\left(18.7^{\circ}\right)$

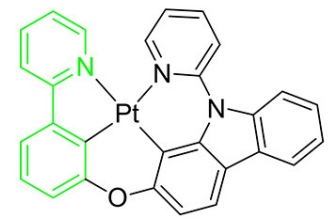

$\mathrm{Pt}($ ppy-O-CbPy)

$\operatorname{Pt}\left(\right.$ ppy) $\left(35.3^{\circ}\right)$

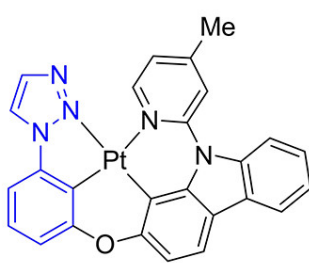

$\mathrm{Pt}(1-p t z-\mathrm{O}-\mathrm{CbPy}-\mathrm{Me})$

Pt(1-ptzMe) $\left(20.8^{\circ}\right)$

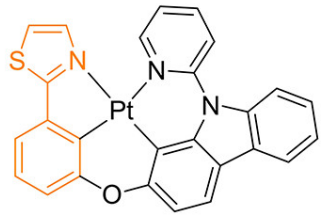

$\mathrm{Pt}($ pthz-O-CbPy)

$\operatorname{Pt}\left(\right.$ pthz) $\left(34.0^{\circ}\right)$

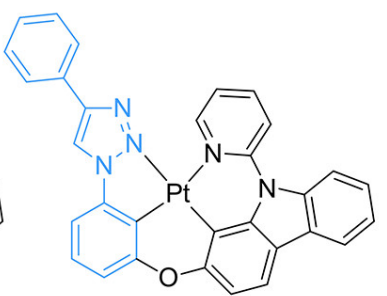

$\mathrm{Pt}(\mathrm{Ph}-1-p t z-\mathrm{O}-\mathrm{CbPy})$

$\mathrm{Pt}\left(1-\right.$ ptzPh) $\left(22.8^{\circ}\right)$

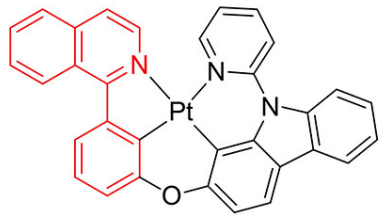

$\mathrm{Pt}($ piq-O-CbPy)

$\operatorname{Pt}\left(\right.$ piq) $\left(41.1^{\circ}\right)$

Fig. 4 Chemical structures of tetradentate Pt(II) complexes. Adapted with permission from Ref. 146 Copyright 2020 American Chemical Society.

involves solving the time-dependent Schrödinger equation along multiple degrees of freedom. This becomes extremely expensive for large molecular systems unless strict selection criteria is imposed on the number of active modes to study. Multiconfiguration time-dependent Hartree (MCTDH) ${ }^{153}$ is a popular method where the time-dependent Schrödinger equation is solved by expanding the nuclear functions into a time-dependent basis set, with timedependent co-efficients

$$
\Psi\left(Q_{1}, \ldots, Q_{f}, t\right)=\sum_{j_{1}=1}^{n_{1}} \cdots \sum_{j_{f}=1}^{n_{f}} A_{j_{1} \ldots j_{f}}(t) \prod_{k=1}^{f} \phi_{j_{k}}^{(k)}\left(Q_{k}, t\right)
$$

$Q_{i}$ are the nuclear coordinates, $A_{j_{1} \ldots j_{f}}$ are the MCTDH expansion coefficients and $\phi_{j_{k}}^{(k)}$ are the $n_{k}$ expansion functions for each degree of freedom $k$, known as the single particle functions. This method has the advantage that the time-dependent basis set ensures convergence with fewer basis functions. A more sophisticated version of the MCTDH method is the multi-layer variant, also known as ML-MCTDH, 154 which has been used to study systems comprising over 1000 degrees of freedom. These approaches combines multiple degrees of freedom of the system under one single particle function, therefore reducing the effective number of degrees of freedom significantly. A more detailed discussion on these approaches are given in Ref. 155

In order to overcome the requirement of grid-based nuclear dynamic methods to pre-compute the potential energy surface, 

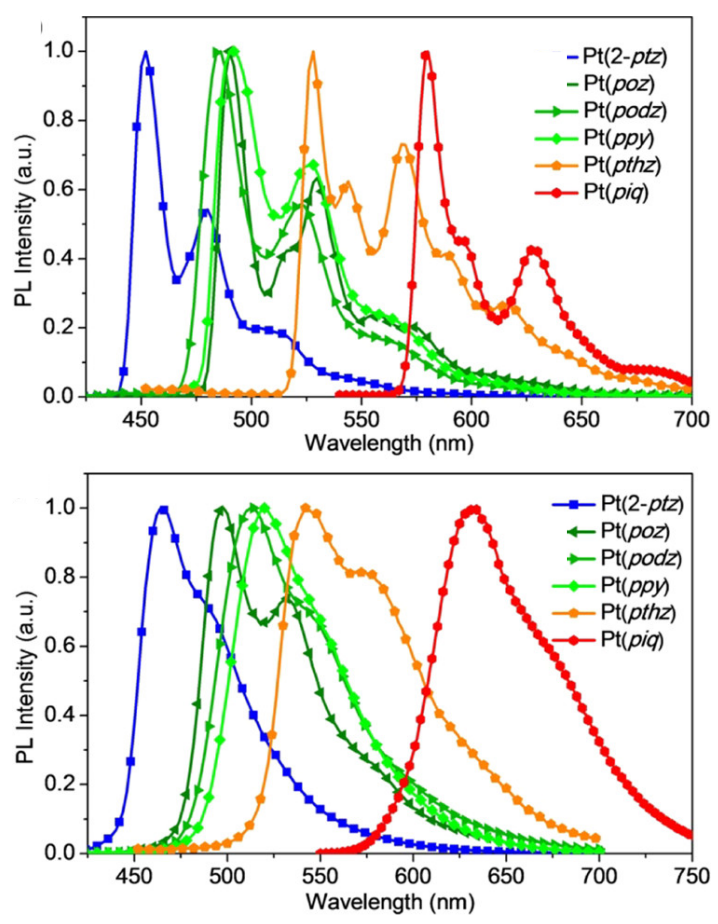

Fig. 5 Photoluminescence spectra of tetradentate $\mathrm{Pt}(\mathrm{II})$ complexes shown in Fig. 4 at 77K (top) and room temperature (bottom). Adapted with permission from Ref. 146 Copyright 2020 American Chemical Society

on-the-fly dynamics has been developed. A wide range of onthe-fly methods are available ranging from fully quantum approaches such as mixed quantum-classical approaches like trajectory surface hopping (TSH). The electronic properties are computed at each time step, allowing for simulations to be performed in the full nuclear configuration space. Significant advances within this framework have been made by González et al., where they have taken into account SOC and non-adiabatic coupling to study the rate of ISC.69/70/73/76 The surface hopping formalism has recently been used in conjunction with the linear vibronic coupling method. 156 The combined approach is computationally faster in comparison to on-the-fly dyanmics and allows for inclusion of all degrees of freedom as opposed to quantum dynamics, without introducing any further additional approximations. A more advanced use of on-the-fly trajectory approaches is based on Gaussian wavepackets instead of independent point trajectories. 157,159 A detailed description of these dynamic methods can be found in the recent review by Penfold et al. 160 We therefore restrict our discussion of ISC to the perturbation theory-based methods.

In the limit of the coupling between the initial and final states involved in ISC being much smaller than the adiabatic energy difference, the rate of ISC can be described by perturbation theory. The common starting point is to approximate the initial and final state potentials by harmonic oscillators having vibrational frequencies, $\Omega_{i}$ and $\Omega_{f}$, respectively. For electronic transitions between the initial $\left(\mathbf{Q}_{\mathbf{i}}\right)$ and final $\left(\mathbf{Q}_{\mathbf{f}}\right)$ potential energy surfaces, the Duschinsky transformation connects the two as,

$$
\mathbf{Q}_{\mathbf{f}}=\mathbf{J} \mathbf{Q}_{\mathbf{i}}+\mathbf{D}
$$

where $\mathbf{J}$ and $\mathbf{D}$ are the Duschinsky matrix accounting for the rotation of the normal coordinates and the displacement vector between the two potentials, respectively. For transitions preserving symmetry, the Duschinsky matrix is block diagonal and the displacement vector has only non-zero elements for totally symmetric modes. It is also worth mentioning here that Eq. 20 is only an approximate way of looking at the electronic transition and is suitable only for cases with minimal change in geometry during the transition. This can be traced back to Eckart condition, which states that two electronic states with different equilibrium geometries and normal modes gives rise to two distinct sets of internal coordinates. As such, for large-amplitude motion as for instance is the case in low-frequency bending modes, Eq20 may not be adequate and the use of different Cartesian normal coordinates or curvilinear/internal coordinates are preferable.161|162

\subsection{Qualitative Rules}

When the SOC integral between the initial and final states is much smaller than the adiabatic energy gap, a qualitative analysis of the rate of ISC $\left(k_{I S C}\right)$ is possible by starting from the "Fermi's Golden Rule" approximation.

$$
k_{I S C}=\frac{2 \pi}{\hbar} \sum_{f}\left|\left\langle\Psi_{f}\left|\hat{H}_{S O}\right| \Psi_{i}\right\rangle\right|^{2} \delta\left(E_{i}-E_{f}\right)
$$

$\Psi_{i}$ and $\Psi_{f}$ are the molecular wavefunctions of the initial and final states including both electronic and vibrational contributions. $\hat{H}_{S O}$ is the spin-orbit Hamiltonian describing the coupling between the two states. The conservation of molecular energy is ensured by the $\delta$ function. Under the approximation that the SOC only depends on the electronic part of the wavefunction, the electronic $(\psi)$ and vibrational $(v)$ contributions are separable and can be rewritten as:

$$
k_{I S C}=\frac{2 \pi}{\hbar} \sum_{f}\left|\left\langle\psi_{f}\left|\hat{H}_{S O}\right| \psi_{i}\right\rangle\right|^{2} \sum_{k}\left|\left\langle v_{f k} \mid v_{i a}\right\rangle\right|^{2} \delta\left(E_{i a}-E_{f k}\right)
$$

As is evident from Eq. 22, the rate of ISC depends on two contributions: (i) purely electronic SOC and (ii) the vibrational density of states. These terms have enabled the qualitative estimation of the rate of ISC.

\subsubsection{El-Sayed's Rule}

El Sayed's rule states that the rate of ISC is larger when the electronic states involved in the non-radiative transition are of different orbital symmetry with respect to the molecular plane of reflection, i.e. transitions must be accompanied by a change in angular momentum. 163 It thus predicts that the rate of ISC is larger for transitions of ${ }^{1}\left(\pi, \pi^{*}\right) \rightsquigarrow^{3}\left(n, \pi^{*}\right)$ type than for ${ }^{1}\left(\pi, \pi^{*}\right) \rightsquigarrow^{3}\left(\pi, \pi^{*}\right)$ (and vice versa). This rule is based on the electronic part of Eq. 22 and the subsequent single-electron nature of SOC operator. However, this rule fails when the two electronic states are close in energy.

El-Sayed's rule was originally discussed in the context of purely 
organic chromophores. Angular momentum operators are purely imaginary and do not have diagonal matrix elements in a basis of real functions. As illustrated in Fig. 6, considering a molecule in the $y z$ plane, the $l_{z}$ operator couples the out-of-plane $\pi$ or $\pi^{*}$ type $p_{x}$ orbitals with the non-bonding in-plane $p_{y}$ orbitals while the spin operator $s_{z}$ changes the sign of the electron spin function, thus transforming the $M_{S}=0$ singlet state to a $M_{S}=1$ triplet state and vice versa.

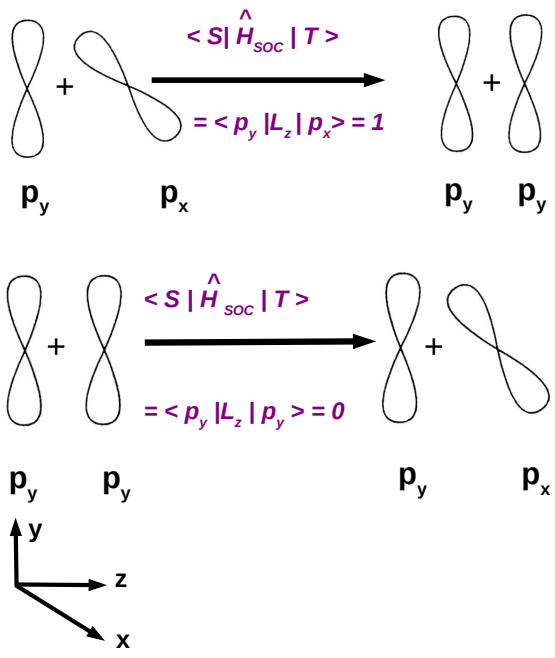

Fig. 6 Orbital rotations upon spin-orbit coupling demonstrated using $p$ orbitals.

The situation is more complicated for transition metal complexes. Naively, one can say that El-Sayed's rule is generally valid due to the $Z_{\text {eff }}^{4}$ dependence of SOC matrix elements. The SOC will be largest for transitions between states with d-electron contributions. Crystal field splitting effects play a crucial role towards the contribution of the d-electron to the MLCT state involved in ISC. For instance, in $\mathrm{Au}(\mathrm{I})$ complexes with metal-to-ligand charge transfer states, crystal field splitting is larger than for analogous $\mathrm{Cu}(\mathrm{I})$ complexes, and thus the delectron contribution to the lowlying excited state is smaller for the former, leading to smaller SOC.

Despite the rule being relatively straight forward, it is based solely on electronic states of a single character. Mixing between states of different orbital character leads to a breakdown of the rule. Moreover, vibronic interactions are known to enhance ISC for El-Sayed forbidden transitions. 160

\subsubsection{Energy Gap Law}

The sum-over vibrational states part of Eq. 22 is commonly referred to as vibrational density of states. The energy separation between the two states undergoing a transition guides the rate of ISC under two limiting situations: the weak coupling and strong coupling cases, as outlined by Jortner and Englman. ${ }^{164}$ In the weak coupling limit, the change in normal mode coordinates are small, and thus the transition probability depends exponentially on the adiabatic energy difference. Therefore, the smaller the energy gap, the larger is the rate of ISC. On the other hand, for the strong coupling limit, there occurs a larger displacement in co- ordinates so that the potential energy surfaces of the two states intersect. Under such circumstances, a higher energy gap usually corresponds to a lower ISC probability. Moreover, the transition probability follows a Gaussian decay with respect to the energy gap and reorganization energy.

\subsection{Franck-Condon Approximation}

A Taylor expansion of Eq. 22 about a chosen reference point $\left(q_{0}\right)$ gives a direct $\mathrm{SOC}$ term,

$$
k_{I S C}^{\text {direct } / \mathrm{FC}}=\frac{2 \pi}{\hbar}\left|\left\langle\psi_{f}\left|\hat{H}_{S O}\right| \psi_{i}\right\rangle\right|_{q_{0}}^{2} \sum_{k}\left|\left\langle v_{f k} \mid v_{i a}\right\rangle\right|^{2} \delta\left(E_{i a}-E_{f k}\right)
$$

a mixed contribution coming from both Franck-Condon and Hertzberg-Teller terms

$$
\begin{aligned}
k_{I S C}^{\text {mixed }}= & \frac{4 \pi}{\hbar} \Re\left(\langle \psi _ { f } | \hat { H } _ { S O } | \psi _ { i } \rangle | _ { q _ { 0 } } \sum _ { k } \left(\left\langle v_{f k} \mid v_{i a}\right\rangle\right.\right. \\
& \left.\left.\times\left.\sum_{\alpha} \frac{\partial\left\langle\psi_{f}\left|\hat{H}_{S O}\right| \psi_{i}\right\rangle}{\partial q_{\alpha}}\right|_{q_{0}}\left\langle v_{f k}\left|q_{\alpha}\right| v_{i a}\right\rangle \delta\left(E_{i a}-E_{f k}\right)\right)\right)
\end{aligned}
$$

and a pure Herzberg-Teller expression,

$$
\begin{aligned}
k_{I S C}^{\mathrm{HT}}= & \frac{2 \pi}{\hbar} \Re\left(\sum _ { k } \left(\left.\sum_{\alpha} \frac{\partial\left\langle\psi_{f}\left|\hat{H}_{S O}\right| \psi_{i}\right\rangle}{\partial q_{\alpha}}\right|_{q_{0}}\left\langle v_{f k} \mid q_{\alpha} v_{i a}\right\rangle\right.\right. \\
& \left.\left.\times\left.\sum_{\beta} \frac{\partial\left\langle\psi_{f}\left|\hat{H}_{S O}\right| \psi_{i}\right\rangle}{\partial q_{\beta}}\right|_{q_{0}}\left\langle v_{f k} \mid q_{\beta} v_{i a}\right\rangle \delta\left(E_{i a}-E f k\right)\right)\right) .
\end{aligned}
$$

The reference point $q_{0}$ is generally chosen to be the equilibrium geometry of the initial state or the crossing point of the two potential energy surfaces. Higher-order terms of the Taylor series expansion are usually negligible. It is further possible to compute the rate of ISC for transition to the individual levels of the triplet states. However, this involves going beyond first-order perturbation theory and requires knowledge about the composition of the triplet sub-states, and no such study has been performed either theoretically or experimentally.

Generally, the rate of ISC is approximated by the direct term (Eq. 23). However, under certain circumstances, it is essential to go beyond the Franck-Condon approximation and include higher order terms as in Eq. 24 and 25 . Higher-order terms of the Taylor series expansion accounts for vibronic SOC contributions. 160 SOC matrix elements are complex valued, and only the real parts contribute to ISC, which is an observable quantity.

Focusing only on the direct contribution towards the rate of ISC, the task in hand boils down to approximating the Dirac $\delta$ distribution. Various methods have been developed to this end. These methods use one of two strategies for integration, either an integration grid over the energy domain or over the time domain. We discuss these methods in further detail in the following sections.

\subsubsection{Time-independent methods}

Toniolo and Persico proposed to replace the Dirac $\delta$ function in Eq. 23 by a step function of finite width. $\frac{165}{1}$ Their approach in- 
volved calculating the Franck-Condon factors and the vibronic couplings within a energy range. Using the Condon approximation, the vibrational part of the rate is thus obtained by summing over the Franck-Condon factors of all states in the energy interval $2 \eta$.

$$
k_{I S C}=\frac{\pi}{\hbar \eta} \sum_{f}\left|\left\langle\psi_{f}\left|\hat{H}_{S O}\right| \psi_{i}\right\rangle\right|_{q_{0}}^{2} \sum_{\left\{k|\eta>| E_{i a}-E_{f k} \mid\right\}}\left|\left\langle\left\{v_{i a}\right\} \mid\left\{v_{f k}\right\}\right\rangle\right|^{2}
$$

The applicability of this approach can be extended through the use of efficient pre-screening of the Franck-Condon factors, sum rules and mode selections. $166-168$ The limitations of this method lies in the fact that the computational cost grows significantly with increasing number of normal modes and/or an increase in adiabatic energy separation between the states, both of which control the vibrational density of states (VDOS). Care must also be taken when choosing the finite step width. Moreover, the energy grid integration protocol gives a thorough insight into the vibrational modes contributing to the non-radiative transition.

Another time-independent approach is to consider the hightemperature limit and compute the transition rate as a sum over Boltzmann-weighted rate constants $k_{I S C}^{k}$, using a population distribution multiplier

$$
k_{I S C}^{k}=\frac{2 \pi}{\hbar} \sum_{f} \mid\left\langle\psi_{f}\left|\hat{H}_{S O}\right| \psi_{i}\right\rangle \|_{q_{0}}^{2} F^{k}\left(E_{i k}\right)
$$

where the Franck-Condon-weighted density of states $\left(F^{k}\right)$ at room-temperature can be estimated from Marcus-Levich-Jortner theory as 169

$$
F^{k}=\left(4 \pi \lambda k_{B} T\right)^{-1 / 2} \sum_{n} \exp \left(-S_{k}\right) \frac{S_{k}^{n}}{n !} \exp \left(\frac{-\left(\Delta E_{i f}+n \hbar \omega_{k}+\lambda\right)^{2}}{4 \pi k_{B} T}\right)
$$

$\Delta E_{i f}$ is the energy gap between the two states at their equilibrium geometries and $\omega_{k}$ the frequency of an effective normal mode involved in the ISC. The dimensionless Huang-Rhys factor $\left(S_{k}\right)$ is given by

$$
S_{k}=\frac{\omega_{k} \Delta Q_{k}^{2}}{2 \hbar}
$$

$\Delta Q_{k}$ is the shift in equilibrium position between the two states. In Marcus theory, the reorganization energy $\lambda$ empirically accounts for solvent-induced relaxations and low-frequency vibration mode contributions. As is apparent from Eq. 28 and 29, the rate of ISC depends strongly on the energy separation and change in geometry. Hence, it is advisable to use accurate methods for geometry optimizations and frequency calculations, while a more approximate method for computing SOC can be used without including much error.

\subsubsection{Time-dependent methods}

In the Heisenberg picture, $\frac{170}{1}$ the need to explicitly calculate the Franck-Condon VDOS can be circumvented by replacing the Dirac $\delta$ function by the corresponding Fourier integral

$$
\delta\left(E_{i}-E_{f}\right)=\frac{1}{2 \pi} \int_{-\infty}^{\infty} e^{i\left(E_{i}-E_{f}\right) t / \hbar} d t
$$

Employing properties of conjugation and resolution of identity, the rate of ISC can be expressed as

$$
k_{I S C}=\frac{1}{\hbar} \int_{-\infty}^{\infty}\left\langle\psi_{i} v_{i a}\left|\hat{H}_{S O}(0) \hat{H}_{S O}(t)\right| \psi_{i} v_{i a}\right\rangle d t
$$

Eq. 31 is exact up to first order in time-dependent perturbation theory, and the spin-orbit coupling operator in the Heisenberg picture is given by

$$
\hat{H}_{S O}(t)=e^{i \hat{H}_{0} t / \hbar} \hat{H}_{S O} e^{-i \hat{H}_{0} t / \hbar}
$$

The calculation of rate constants using this protocol is not restricted to harmonic potentials nor the Condon approximation for the spin-orbit matrix elements. The evaluation of the autocorrelation function nevertheless becomes simpler. The autocorrelation function $(G(t))$ was first established by Kubo and Lax, $, 171,173$ and has been widely used to compute non-radiative rate constants both analytically and numerically $[61|161| 174 \mid 1755$ Etinski et al. employed the correlation function to arrive at an analytical expression for the rate of ISC within the Condon approximation for vibrationally cold molecules as

$$
k_{I S C}=\frac{1}{\hbar}\left|\left\langle\psi_{f}\left|\hat{H}_{S O}\right| \psi_{i}\right\rangle\right|^{2} \int_{-\infty}^{\infty} G(t) e^{i\left(E_{i}-E_{f}+0.5 \operatorname{tr}\left(\Omega_{i}\right)\right) t / \hbar} g(t) d t
$$

For the precise definition of the different quantities appearing in this equation, we refer to Ref. 176. A carefully chosen Gaussiantype damping factor $\left(g(t)=\exp \left(-\eta t^{2}\right)\right)$ can be used to accelerate the convergence of the correlation function. This can be seen as accounting for all the additional possible damping that the vibronic states undergo, such as vibrational relaxation, radiative transition and coupling between normal modes. Recently, we modified this approach, incorporating the Boltzmann distribution function to calculate rate of ISC both at finite temperatures and in the low-temperature limit, $\underline{174}$

$$
k_{I S C}=\frac{2}{Z}\left|\left\langle\psi_{f}\left|\hat{H}_{S O}\right| \psi_{i}\right\rangle\right|^{2} \int_{0}^{\infty} \sqrt{\sqrt{\left(U^{2}+V^{2}\right)}} e^{K_{1}} \cos \left(\frac{\theta}{2}+K_{2}\right) d t
$$

In the above simplified expression, we have used the same notation as in Ref. 174.

An alternative procedure is to use the first- and second-order cumulant probability distribution for the time-ordered exponential functions 109

$$
k_{I S C}=4 \pi\left|\left\langle\psi_{f}\left|\hat{H}_{S O}\right| \psi_{i}\right\rangle\right|^{2} e^{-\kappa_{2}^{\mathrm{R}, \mathrm{TI}}} \times \int_{0}^{\infty} \cos \left(\kappa_{1} t+\kappa_{2}^{\mathrm{Im}}\right) e^{-\kappa_{2}^{\mathrm{R}, \mathrm{TD}}} d t
$$

The same notations are used in Eq. 35 as in Ref. 109. Furthermore, Etinski et al. ${ }^{[61}$ have derived a short-time expression which avoids numerical integration when calculating the rate of ISC . Both the exact expression and the cumulant expansion perform well and neither of them outperforms the other.

The methods described above are all computed using standard numerical integration procedures. Alternatively, analytical solutions are also possible by appropriately modelling the Hamiltonian by analytic functions, which are then evaluated by recursion formulas. 161$] 177+179$ The model Hamiltonian in use is the simple harmonic oscillator. In this case, the calculations are performed in 
the Fourier-transformed frequency space. Pioneering work in this field has been done by Borrelli and Peluso, with most application focusing on energy transfer mechanisms. $175[180 \mid 181$

\subsection{Illustrative examples}

Calculations of the rate of ISC within the Franck-Condon domain has been successfully used for various transition metal complexes. $109182-186$ Here we discuss representative $5 \mathrm{~d}$ transition metal systems, with the aim to generate a better understanding of ISC processes based on the interplay of non-equilibrium excitedstate dynamics and structural changes, and also the finer details brought to light by a detailed analysis of the effects of Duschinsky mixing.

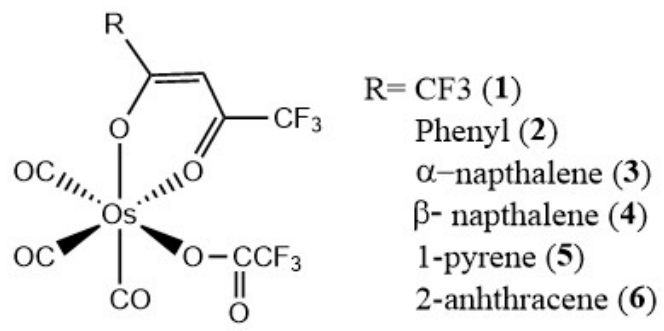

Fig. 7 Chemical structure of Os(II) complexes. Adapted with permission from Ref. 128 Copyright 2007 Royal Society of Chemistry.

Table 1 Triplet lifetime of Os(II) complexes, the extent of mixing between $\pi \pi^{*}$ and MLCT character and luminescence in $\mathrm{CH}_{2} \mathrm{Cl}_{2}$ at room temperature. $\mathrm{P}$ and $\mathrm{F}$ denotes phosphorescence and fluorescence, respectively. Data taken from Ref. 128/187

\begin{tabular}{llll}
\hline Complex & $\tau_{I S C}=\frac{1}{k_{I S C}}$ & MLCT in $\pi \pi^{*}$ & emission wavelength \\
\hline Os-1 & $150 \mathrm{fs}$ & $11 \%$ & $545 \mathrm{~nm}(\mathrm{P})$ \\
Os-2 & $0.48 \mathrm{ps}$ & $4.0 \%$ & $520 \mathrm{~nm}(\mathrm{P})$ \\
Os-3 & $3.64 \mathrm{ps}$ & $1.3 \%$ & $560 \mathrm{~nm}(\mathrm{P})$ \\
Os-4 & $6.71 \mathrm{ps}$ & $1.0 \%$ & $540 \mathrm{~nm}(\mathrm{P})$ \\
Os-5 & $120 \mathrm{ps}$ & $0 \%$ & $550 \mathrm{~nm}(\mathrm{~F}) \& 690 \mathrm{~nm}(\mathrm{P})$ \\
Os-6 & $2.1 \mathrm{~ns}$ & $0 \%$ & $575 \mathrm{~nm}(\mathrm{~F})$ \\
\hline
\end{tabular}

In order to demonstrate structure-dynamic relationships we consider a set of analogous Os(II) complexes (Fig. 7), which exhibits changes in the luminescence behavior from purely phosphorescent (Os 1-4) to dual emissive (Os-5) to purely fluorescent (Os-6) $128 / 187$ These varying features are guided by the rate of ISC. The change in character of the lowest excited state dictates this behavior. The polyaromatic pendant introduces $\pi$ conjugation at the $\beta$-diketonate, reducing the energy separation between the $S_{1}$ and $T_{1}$ states. Additionally, the very fast ISC for Os-1, can be rationalized from it having the highest MLCT character in the $\pi \pi^{*}$ orbital. (Table 1) The trend in the rate of ISC in these complexes follows the percentage mixing of $\pi \pi^{*}$ by MLCT character.

To further reinforce this concept, let us look at a pair of arylacetylide $\mathrm{Au}$ (I) (Au-1) and $\mathrm{Au}$ (III) (Au-2) complexes (Fig. 8). Experimentally, the rates of ISC are found to be $0.75 \times 10^{7} \mathrm{~s}^{-1}$ for Au-1 and $7.57 \times 10^{10} \mathrm{~s}^{-1}$ for Au-2. The large difference is explained in terms of El-Sayed's rule and the energy gap law. Fig. 9 shows that the available pathways for ISC are from $S_{1}$ state to

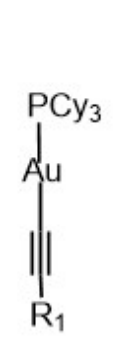

(1a) (1b)
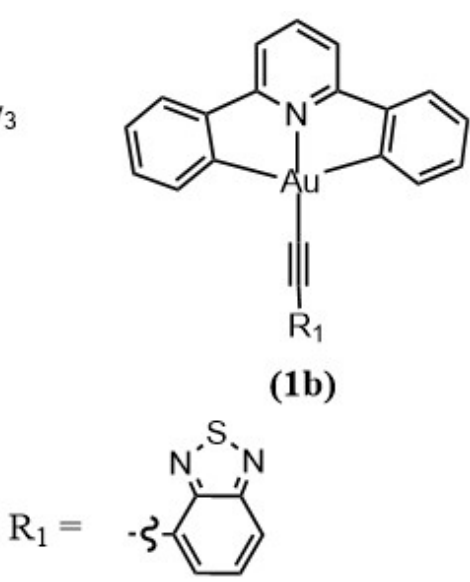

Fig. 8 Chemical structure of $\mathrm{Au}(\mathrm{I})$ and $\mathrm{Au}(\mathrm{III})$ complexes. Adapted with permission from Ref. 188 Copyright 2017 Royal Society of Chemistry.

the $T_{2}$ and $T_{4}$ states in Au-1 and to the $T_{4}$ and $T_{5}$ states in Au-2. For Au-1, transition is unfavourable to both of these triplet states as they compete with the $\mathrm{T}_{1}$ state and they all have the same orbital characteristics. In addition, for $T_{4}$, even though one can observe efficient SOC, the energy separation $\left(\Delta E=3180 \mathrm{~cm}^{-1}\right)$ is too large to be overcome by thermal activation. On the other hand, in the Au- 3 complex, facile $S_{1}$ depopulation occurs to the $T_{5}$ state which is both favoured by higher SOC $\left(4100 \mathrm{~cm}^{-1}\right)$ and thermally achievable small energy gaps.

After establishing the success of semi-quantitative approaches, we now turn our attention to quantitative methods for computing rates of ISC. We will consider the photophysics of (acetylacetonato)-bis(1-methyl-2-phenylimidazol)iridium(III) (N966), 108, which has a distinctive dual phosphorescence behavior leading to white light emission. N966 shows dual phosphorescence from both the $\mathrm{T}_{1}$ and $\mathrm{T}_{2}$ states. This anomalous behavior is due to a difference in nature of the $\mathrm{T}_{1}$ and $\mathrm{T}_{2}$ states, ensuring efficient $S_{1} \rightsquigarrow T_{2}$ conversion, but a low rate of IC between the two triplet states. The $\mathrm{T}_{2}$ state is of $\mathrm{d}_{x^{2}-y^{2}} \pi \rightarrow \mathrm{n} \pi$ character whereas the $\mathrm{S}_{1}$ and $\mathrm{T}_{1}$ states are of $d_{x z} \pi \rightarrow p \pi$ character.

As shown in Fig. 10, the smaller singlet-triplet energy gap and higher SOC for $S_{1} \rightsquigarrow T_{2}$ leads to a more favourable ISC than for $S_{1} \rightsquigarrow T_{1}$. However, the computed rate of ISC using the timeindependent Marcus-Levich-Jortner theory predicts a comparably fast rate of ISC for both pathways. The authors attribute this to the Franck-Condon-weighted density of states, which compensates for the smaller SOC. The energy separation thus does not play a significant role as it appears in the decaying exponential term in Eq. 28 .

We next consider a pair of Pt(II) complexes (Fig. 11) containing acetylacetonate and extended cyclometalated 2phenylpyridine (Pt-ppy) and 2-(2/-thienyl)pyridine ligands (Ptthpy). ${ }^{109} 189$ Meticulous variation of Duschinsky rotation parameters gives great insight into the variation in the rates of ISC computed using the time-dependent correlation function and the cumulant expansion-based approach at both room temperature and OK.

The rates (see Table 2) of ISC suggests that in Pt-ppy com- 


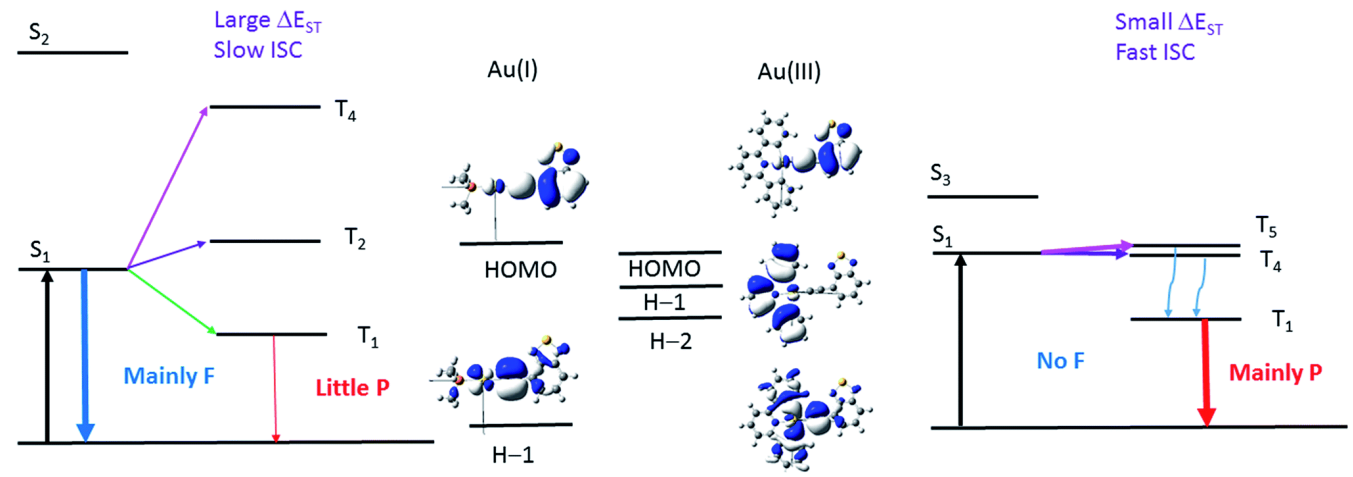

Fig. 9 Illustration of the low-lying singlet and triplet excited states of $\mathrm{Au}(\mathrm{I})$ (left) and $\mathrm{Au}$ (III) (right) complexes that accounts for the different photophysical behavior. The d-orbitals involved in the $T_{2}$ of Au-1 and $T_{4}$ of Au-2 have the same orientations as their respective $S_{1}$ excited state. $\mathrm{F}$ and $\mathrm{P}$ refer to fluorescence and phosphorescence, respectively. Adapted with permission from Ref. 188 Copyright 2017 Royal Society of Chemistry.

Table 2 Photophysical parameters of Pt-ppy and Pt-thpy complexes. Correlation function and cumulant expansion approach based calculations have been performed using 20,000 grid points, damping factor of 2.0 and upper limit of time integration as 20 ps. Data taken from Ref. 109 Energy separation is in $\mathrm{eV}, \mathrm{SOC}$ is in $\mathrm{cm}^{-1}$ and all rates are in $\mathrm{s}^{-1}$. a and $\mathrm{b}$ are for rates computed without $\mathbf{J}$ and $\mathbf{D}$, respectively.

\begin{tabular}{lllll}
\hline Parameters & Temp. $(\mathrm{K})$ & Pt-ppy $S_{1} \rightsquigarrow T_{1}$ & Pt-ppy $S_{1} \rightsquigarrow T_{2}$ & Pt-thpy $S_{1} \rightsquigarrow T_{1}$ \\
\hline$\Delta E_{S T}$ & & 0.55 & 0.08 & 0.80 \\
SOC & 0 & 75.27 & 329.62 & 1.04 \\
$k_{I S C}^{\text {cum }}$ & 0 & $8.60 \times 10^{10}$ & $4.59 \times 10^{13}$ & $5.75 \times 10^{5}$ \\
$k_{I S C}^{\text {corr }}$ & 300 & $7.1 \times 10^{10}$ & $1.07 \times 10^{13}$ & $4.50 \times 10^{5}$ \\
$k_{I S C}^{\text {corr }}$ & 300 & $3.47 \times 10^{10}$ & $5.87 \times 10^{13}$ & \\
$k_{I S C}^{\text {corr }} a$ & 300 & $3.10 \times 10^{10}$ & $5.02 \times 10^{13}$ & \\
$k_{I S C}^{\text {corr }} b$ & $8.51 \times 10^{9}$ & $2.74 \times 10^{12}$ & $6.24 \times 10^{4}$ \\
\hline
\end{tabular}

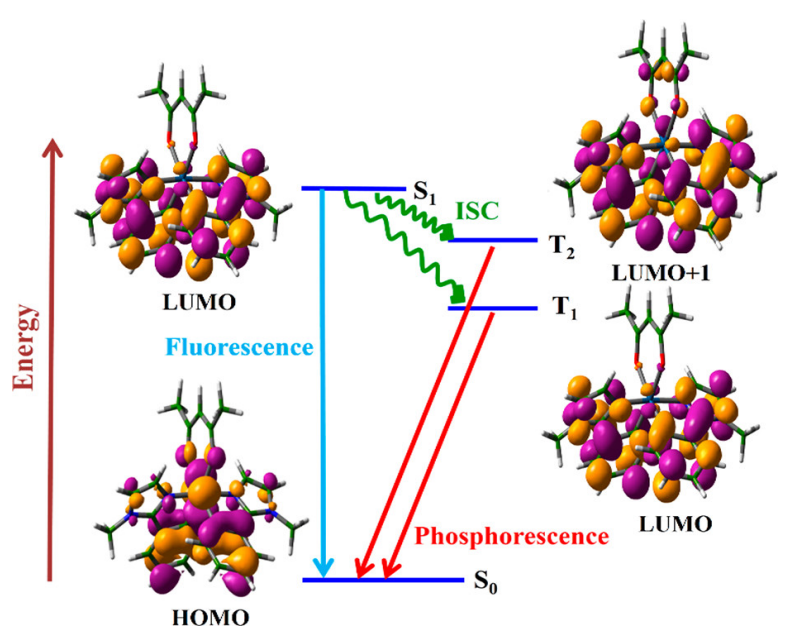

Fig. 10 Jablonski diagram for the photophysical process of N966 complex along with the frontier molecular orbitals involved in the transitions. Adapted with copyright from Ref. 108 Copyright 2017 American Chemical Society.

plexes, there is a rapid depopulation of the singlet-excited state, allowing for radiative emission only from the triplet manifold. In contrast, in Pt-thpy, the rate of ISC enables dual emission. It is worth noticing that the rates calculated using the correlation function at $300 \mathrm{~K}$ is an order of magnitude smaller than that at $0 \mathrm{~K}$. At finite temperatures the excess vibrational energy in the $S_{1}$ state reduced the ISC compared to at $0 \mathrm{~K}$.

We have also calculated the rates without including structural

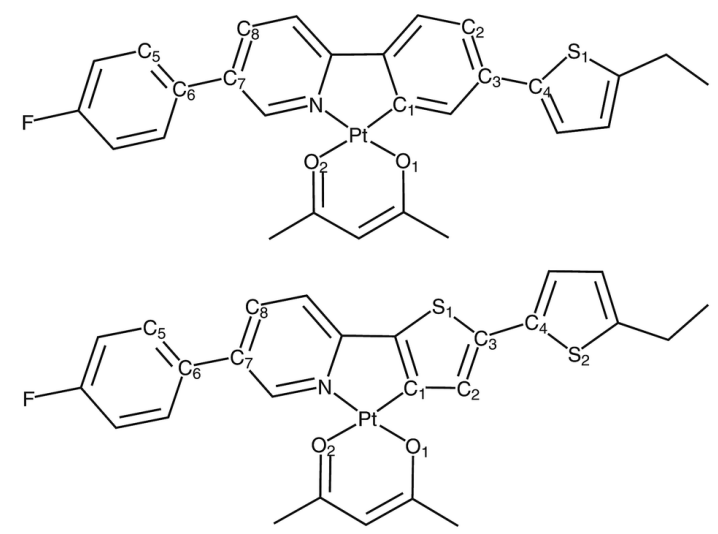

Fig. 11 Chemical structure of Pt-ppy (top) and Pt-thpy (bottom) complexes. Adapted with copyright from Ref. 109 Copyright 2018 Royal Society of Chemistry.

changes, that is, ignoring the $\mathbf{J}$ matrix and $\mathbf{D}$ vector of Eq. 20 The dependence of the displacement vector on the rate of ISC in Eq. 34 occurs through the $K_{1}$ and $K_{2}$ terms. The dependence on $K_{2}$ enters in the cosine term, which varies between \pm 1 . The most prominent feature is that the sign of $K_{1}$ is negative, suggesting that a larger displacement vector contributes less to the net ISC value. In all cases, exclusion of the displacement vector reduces the rate by one order of magnitude.

The Duschinsky rotation matrix (shown in Fig. 13) couples the normal vibrational modes via the off-diagonal elements, enabling ISC transition. In the case of the $S_{1} \rightsquigarrow T_{2}$ transition for the Ptthpy complex, the off-diagonal elements have comparable values 


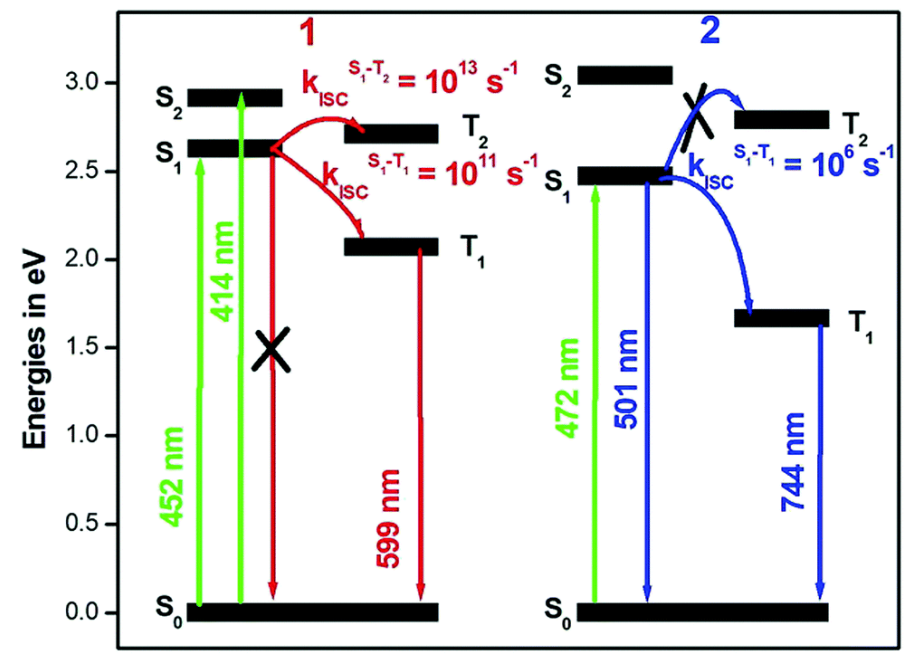

Fig. 12 Modified Jablonski diagram describing the photophysics of Ptppy and Pt-thpy complexes. Adapted with copyright from Ref. 109 Copyright 2018 Royal Society of Chemistry.

to the diagonal elements, and the mixing is confined within a few normal modes. Therefore, neglecting the strong off-diagonal elements leads to a decrease in the rate of ISC by one order of magnitude.

\section{Recent Advances}

During the last 2-3 years, an enormous surge of research interest in the field of non-radiative decay process has been witnessed both at the experimental and the theoretical level. The experimental observations of the breakdown of Hund's rule or the inversion of the lowest singlet and triplet states vis-a-vis barrier free reverse intersystem crossing due to exciton-polariton interaction is ground breaking and, of course demands the development of new theoretical tools to analyze such phenomenon from first principles. $\frac{190}{}$ Harvesting triplet exciton via ISC in transition metal complex, like Platinum(II)-octaethyl-porphyrin (Pt-OEP), has been realized in a Fabry-Perot microcavity due to the formation of hybrid polariton states. 191 It is worth noting that albeit the work of Li et al. 192 is not related to the transition metal complexes, it still deserves a special mention since the combined experiment and multi-reference ab initio calculations suggest that ISC may even take place due to long-range dipole-dipole interactions between the radical fragments produced in a crossedbeam experiment. Recently, Monni et al., 193 have tracked the vibratational coherence transfer during the ultrafast ISC in the tetrakis ( $\mu$ - pyrophosphito)diplatinate(II) complex. According to the authors, the solvent-induced stabilization of the triplet states facilitates the ISC process to the extent that the time taken for the non-radiative spin state transition is much shorter than the dephasing time of the vibrational wave packets. Besides, Mewes et al. 194 have made similar investigation on an another platinum complex, namely, $\left[\mathrm{Pt}(\mathrm{ppy})\left(\mu-\mathrm{tBu}_{2} \mathrm{pz}\right)\right]_{2}$ and found that the structural flexibility of this complex leads to the dephasing of the wave packet formed between the ground and the excited electronic states of the complex before the commencement of the ISC. The role ISC in therapeutics has also been explored a bit in the recent past. The efficient triplet state population gain in $\mathrm{Ru}$, Ir, Pt, Pd and Sn based complexes has immense potential in photodynamic therapy(PDT) and photo- activated chemotherapy, and significant progress has been already been made during the last few years. 195 For example, Phthalocyanine-assembled nanodots act as a triplet photosensitizers that promotes the generation of reactive oxygen species and thus allowing the system to act as a good candidate for PDT.

An impressive progress in the domain of theoretical nonradiative photodynamics has also been made in the past couple of years. Mai and Gonzalez gave an excellent review on the challenges in electronic structure theory and basis function vis-a-vis trajectory-based nuclear dynamics. ${ }^{196}$ The review also focuses on how to accelerate the in-silico photochemical and photophysical investigation on large molecular systems by invoking linear scaling, QM/MM and the Frenkel exciton model. Since the goal of the present perspective is to highlight all types of spin-forbidden transitions, we take the liberty of emphasizing some recent fundamental works that are in line with our objectives. Valentine and $\mathrm{Li} 197$ have recently proposed a new method for calculating the rate of ISC where the spin-orbit coupling is treated variationally and the proposed method has been implemented in the framework of the exact-two component (X2C) method in combination with time-dependent density functional theory. This theoretical approach of calculating the rate of ISC will be particularly important when the strength of the spin-orbit coupling is large. Another new technique for estimating SOC based on El-Sayed's rule has been developed by Krylov and co-workers, 86[198. They generate the natural transition orbitals which allows treating transitions between states with arbitrary spin multiplicities on an equal footing and to visualize and quantitatively estimate the contributions of specific orbital pairs into the overall SOC.

Simulation of ISC dynamics at finite temperature using the thermal Schrödinger equation is challenging although such a thermo-field method is already available for studying nonradiative process like energy transfer. $\frac{199}{19}$ The role of spin-vibronic interaction for the quantitative estimation of the rate of reverse intersystem crossing is shown to be well justified by Kim et al. 200 The advances in trajectory-based dynamics is also quite remarkable. In particular, exploring internal conversion and ISC at the same level using exact factorization of the electron-nuclear wave function makes the generalized coupled-trajectory mixed quantum-classical algorithm very reliable. 201 The use of machine learning to the study of nonadiabatic excited state dynamics is a recent development which reduces the computational costs without compromising the accuracy of the calculations. $\frac{202}{2}$

\section{Practical applications}

The reason for the wide-ranging applicability of transition metal complexes is due to their high photostability, minimal selfquenching, wide range of photo-emission energies, and most importantly the fact that their luminescence properties can be readily modified by the use of a wide variety of ligands of different structural, electronic and coordinating properties.203.205

For these reasons, the development of luminescent transition metal complexes as sensors, organic light emitting diodes 

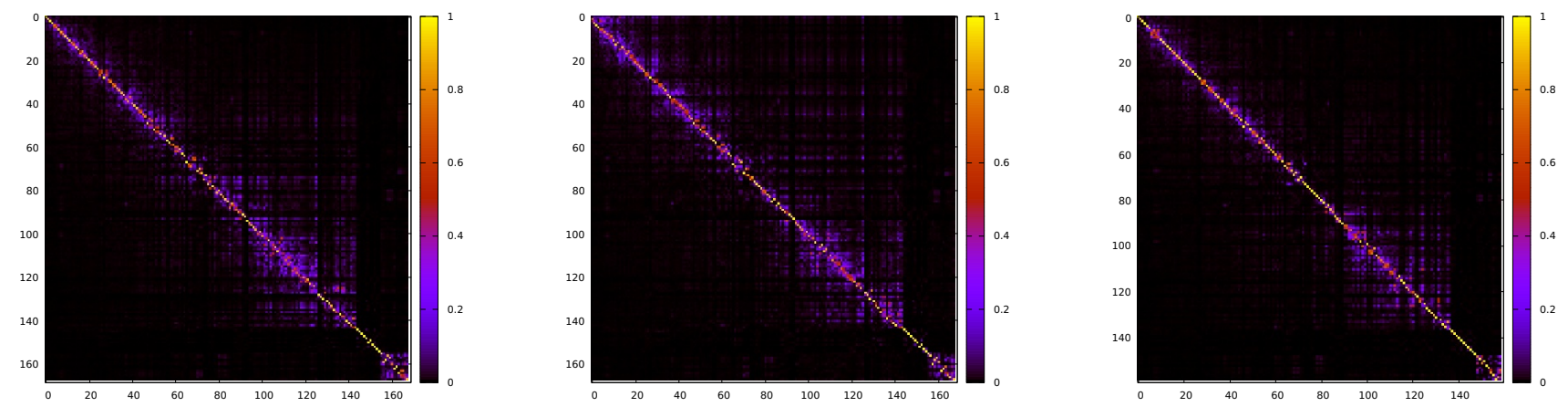

Fig. 13 Absolute value of Duschinsky matrix for $S_{1} \rightsquigarrow T_{1}$ (left) and $S_{1} \rightsquigarrow T_{2}$ (middle) of Pt-ppy and $S_{1} \rightsquigarrow T_{1}$ (right) of Pt-thpy. Taken from Ref. 109

(OLEDs) and dye-sensitized solar cells, has received increasing attention in recent years. Applications of highly phosphorescent transition metal complexes as cellular reagents, such as, biosensors and bioimaging reagents, also serve as a constant source of research. In the following sections, we give some examples of the use of transition metal complexes in OLEDs, light emitting electrochemical cells (LECs) and biological applications.

\subsection{OLEDs}

OLEDs consist of a cathode and an anode, with the region in between comprising of electron and hole carriers, which combine to form excitons. These excitons undergo de-excitation, thereby emitting light. 206 The excitons may either be singlet or triplet in configuration, in the ratio of $1: 3$, as predicted by spin statistics. SOC effects play a crucial role in maximizing the use of all singlet and triplet excitons and thus achieving 100\% internal quantum efficiency, via provision of intense mixing of spin multiplets. Heavy-metal-containing phosphors such as in Ir and Pt complexes can play a key role in OLEDs by efficiently harvesting all the electron-generated excitons, and hence, procuring high efficiency for the OLEDs. 142 203204

This approach has been studied since 1998 and it's first successful commercial application was made in 2003, in the form of phosphorescent red OLEDs. Following this, OLED technology has been under continuous research and improvement, which has led to the extensive use of OLEDs in the development of solid-state lighting (SSL), 207 flat-panel displays and flexible screens. It has also led to red, green and blue (RGB) platforms ${ }^{206}$ of certain efficient phosphorescent dyes, which have been commercialized as displays in small hand-held devices and television sets. Also, white OLEDs have been found to be of immense importance with respect to lighting. 208

Iridium (III) is the most commonly used transition metal in the design of OLEDs. Numerous mono-,bis-cyclometallated homoand hetero-leptic iridium complexes are available, 209210 which can be used as phosphorescent emitters in OLEDs. 211/212 Polymers containing Ir(III) complexes are also considered promising since they offer the appealing combination of a phosphor and a polymer in a single material. Moreover, ancilliary ligands, such as acetylacetonate(acac), picolinate(pic) and bipyridine(bpy) and their structural analogues, offer additional opportunities for tuning the electro-optical properties. For instance, Ir(III) acetylace- tonates, with -OPh as substituent at the cyclometallating ligand, emit a bluish-green color, whereas those with $\mathrm{B}$ (mesithyl) $)_{2}$ as substituent give a red color. ${ }^{213}$ The devices, moreover exhibit excellent power efficiencies $\left(\eta_{P}=26.8-28.6 \mathrm{lmW}^{-1}\right)$ and attain external quantum efficiencies of $\eta_{\text {ext }}=10.3-11.1 \%$.

In order to achieve enhanced thermal stability, increased solubility in solvents and polymer matrices, colour tunability, and high solid-state photoluminescence quantum efficiency, sterically hindered dendrons are often inserted at the periphery of an emitting core. Significant work on phosphorescent Ir(III) complexes with dendronic ligands have been carried out by Burn and Samuel's groups. $214 \mid 215]$ They were able to obtain very high device efficiencies from a series of dendrimer Ir(III) complexes, fastened with 2-ethylhexyloxy terphenyl dendrons, via the site isolation effect of the dendrimers. A maximum external device quantum efficiency of $10.4 \%$ was achieved for a blue electrophosphorescence, highlighting the beneficial role of the dendrons.216|217

More recently, Pt(II), Zn(II), Cu(I) and other transition complexes have been used as materials for OLEDs. 218 221 In the limited scope of this perspective, it is not possible to do justice to the wide range of transition metal complexes which are being explored in the world of OLEDs. Hence, we have restricted our discussion to a general description of design principles and a few specific examples of transition metal complex incorporated in OLEDs.

\subsection{LECs}

Monolayer electroluminescent devices consisting of a luminescent material formed by recombination of ionic charges, sandwiched between two electrodes are regarded as Light-emitting Electrochemical Cells (LECs). The operational mechanism of LECs can be described by two models: the electrodynamical model (ED) 222 and the electrochemical doping (ECD) model.223 Though there are certain differences in the assumptions of the two models, both models agree that the accumulation of ions at the two electrodes results in a sharp drop in the applied potential, resulting in charge reconstitution and hence, triggering light emission, from the phosphorescent material clamped between the electrodes. LECs are easily engineered and their biggest advantage lies in their insensitivity towards the work function of the electrodes, unlike the highly sensitive and complexly structured OLEDs. This allows for the use of air-stable metals such as Au or Ag. 206]224 
Pei et al. 223 first introduced the concept of LECs in 1995, by coalescing an inorganic salt to a blend of a conjugated luminescent polymer and an ionic conductive polymer. Following this pioneering work, Maness et al. ${ }^{225}$ proposed an alternative pathway by incorporating a $\mathrm{Ru}$ (II) ionic transition metal complex (iTMC) as the only active component in the luminescent layer.

However, there is a constraint in color tunability for the Ru(II) chromophores, whose emission band is situated across the orange-red part of the visible spectrum. This hinders their application in lighting and display technologies, which demands extensive colour tunability. The entire color scale can be accessed by using luminescent iTMCs involving different metal centres. For example, in going from $\mathrm{Ru}$ to Ir, enhanced metal-ligand bond strength and ligand field splitting energy promotes better stability of the subsequent complexes, which in turn results in high quantum efficiency and satisfactory photochemical stability. Slinker et al. reported the first example of LECs consisting of a monolayer emitting yellow light and incorporating the Ir iTMC( $\left.\left[\operatorname{Ir}(\mathrm{ppy})_{2}(\mathrm{dtb}-\mathrm{bpy})\right]\left[\mathrm{PF}_{6}\right]\right), 226$ which displays a photoluminescence quantum yield of $23.5 \%$ in oxygen-free acetonitrile solution.

Several strategies have been adopted to design LECs with emissions across the entire visible spectrum. 224 Ir(III) complexes have commonly used for this purpose, with light-emitting complexes from red $\left[\operatorname{Ir}(\mathrm{ppy})_{2}(\mathrm{qIbi})\right]\left[\mathrm{PF}_{6}\right]$, to yellow $\left[\operatorname{Ir}(\mathrm{ppy})_{2}(\mathrm{dtb}-\right.$

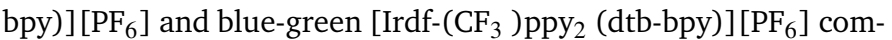
plexes. 224

In short, LECs have made notable progress over the past few years, which when combined with it's advantages over OLEDs, enable us to consider LECs as affordable, versatile and energyefficient flat light sources.

\subsection{Biological Applications}

The use of phosphorescent transition metal complexes in biology encompasses an extensive area of applications as sensing agents, bioimaging probes, therapeutic agents and theranostic agents. 227

\subsubsection{Role as Labelling Agents}

DNA or protein intercalation enables the charged transition metal complexes to bind covalently or noncovalently to the biological substrate. 228 There is usually a difference between the luminescence exhibited by the free substrate and that by the bound molecule, resulting from changes in the rigidity and hydrophobicity of their environment. This facilitates monitoring of bioconjugation reactions and hence, the transition metal complexes can function as labelling agents for luminescence imaging. For example, Yu et al. reported the reduction in cytotoxicity and photobleaching of two cationic Ir(III) complexes into the cytoplasm of cervical cancer cells compared to regular dyes. 229

\subsubsection{Role as Cellular Probes}

Treatment of cancer normally involves the embedding of metal complexes into DNA via a host-guest relationship (with DNA acting as the host), resulting in inhibition of DNA replication, which in turn facilitates cell death.227

Extensive studies of the photophysically enriched Ru(II)- diimine complexes have revealed that, although, [Ru(bpy)3 $]^{2+}$ did not show any enhancement in luminescence in the presence of duplex DNA, the more extended $[\mathrm{Ru}(\mathrm{bpy}) 2(\mathrm{dppz})]^{2+}, 230$ and $[\mathrm{Ru}(\mathrm{phen}) 2(\mathrm{dppz})]^{2+, 231}$ complexes displayed intensely amplified luminescence.This amplified luminescence can be attributed to the protection of the dppz moiety from solvent quenching upon intercalation into DNA, which ultimately triggered an effective emission from the MLCT excited state of the complex. Application of fluorescence lifetime imaging microscopy (FLIM) ${ }^{232}$ to the $\Delta$ and $\Lambda$ isomers of Ru(II)-dppz complexes in live and fixed cells revealed that these complexes can serve as potential probes for biomolecular binding and cellular microenvironments. 233

\subsubsection{Role as Oxygen Sensors}

Molecules with triplet ground state, like oxygen, can productively quench the phosphorescence of Ir(III) polypyridine complexes, thereby making them excellent oxygen sensors. They fulfill the necessary requirements of high emission quantum yields, long emission lifetimes, high sensitivity, high reversibility and fast response time, to be ideal oxygen sensors. Huynh et al. ${ }^{234}$ produced two luminescent Ir(III) complexes $\left[\operatorname{Ir}(\mathrm{ppy})_{2}(\mathrm{CN})_{2}\right]$ and $\left[\operatorname{Ir}(\mathrm{ppy})_{3}\right]$ as oxygen sensors in poly $((\mathrm{n}$ butylamino)thionylphosphazene) (nBuPTP) matrixes. A reduction in emission intensity and lifetimes in the presence of oxygen, with a linear SternâĂŞ-Volmer relationship, was displayed by $\mathrm{nBuPTP}$ polymer films containing these complexes.

Owing to these multiple roles successfully played by luminescent transition metal complexes, of which only a few have been discussed above, phosphorescent transition metal complexes have a wide use as dual-function metal complexes (possessing both diagnostic and therapeutic qualities in a single entity) in cancer theranostics.

\section{Summary}

We have in this perspective focused on the intersection of photophysical processes and transition metal complexes. Transition metal complexes are an interesting group of compounds with unique structure-bonding and luminescence properties. The metal centers are capable of bonding to a wide range of ligands and with varying coordination numbers. The formation and substition of metal-ligand bonds are easily accomplished. This allows their photophysical properties to be changed by altering the ligand numbers and types. The photophysics is mostly controlled by the characteristic large nuclear charge of the metal atom, which in turn gives rise to SOC between the singlet and triplet manifolds. The loss of pure spin states opens up new deactivation pathways, with ISC and phosphorescence being the two most important one. We have in this perspective discussed the theoretical foundation as well as given examples of three central quantities determining the photophysics of transition metal complexes, namely, SOC, ISC and phosphorescence.

The demand for computational techniques to unravel the complexities behind photoluminescence spectroscopies has led to efficient implementations of these properties in quantum-chemical softwares packages such as DALTON program 235, QChem software $^{236}$, Gaussian 237, DFT/MRCI ${ }^{238}, \mathrm{ADF}^{239}$, SPOCK ${ }^{136}$ and 
ReSpect 240 A number of programs can be used to perform frequency calculations and Franck-Condon analysis.237|239|241|242 However, the choice for computing rates of ISC are limited and are more often stand-alone codes developed by individual groups. Even though we have not discussed quantum dynamic methods explicitly, we note that SHARC and MCTDH are the two dominant programs in use for this purpose. $\frac{153 \mid 243}{1 n}$ order to appropriately utilize these computational tools, an overall understanding of the theory is essential, and hopefully this contributed to it.

The novelty of transition metal complexes are far and wide. A variety of applications in the fields of opto-electronics and photobiology have been discussed. Ir(III) complexes are among the most popular candidates for this purpose. The field is growing rapidly and remains a hot research topics.

\section{Conflicts of interest}

There are no conflicts to declare.

\section{Acknowledgements}

T. M. acknowledges support from the Marie Skłodowska-Curie European Training Network "COSINE-COmputational Spectroscopy In Natural sciences and Engineering", Grant Agreement No. 765739. P. K. thanks the Council of Scientific and Industrial Research (CSIR) for granting him the Junior Research Fellowship. KR acknowledges support from the Research Council of Norway through a Centre of Excellence Grant (Grant No. 262695).

\section{Notes and references}

1 M. Fumanal, E. Gindensperger and C. Daniel, J. Phys. Chem. Lett., 2018, 9, 5189-5195.

2 B. A. McClure and J. J. Rack, Inorg. Chem., 2011, 50, 75867590.

3 Z. Guo, P. G. Giokas, T. P. Cheshire, O. F. Williams, D. J. Dirkes, W. You and A. M. Moran, J. Phys. Chem. A, 2016, 120, 5773-5790.

4 G. B. Shaw, C. D. Grant, H. Shirota, E. W. Castner, G. J. Meyer and L. X. Chen, J. Am. Chem. Soc., 2007, 129, 21472160.

5 N. Huse, T. K. Kim, L. Jamula, J. K. McCusker, F. M. F. de Groot and R. W. Schoenlein, J. Am. Chem. Soc., 2010, 132, 6809-6816.

6 R. M. van der Veen, A. Cannizzo, F. van Mourik, A. Vlček and M. Chergui, J. Am. Chem. Soc., 2011, 133, 305-315.

7 J. E. Monat and J. K. McCusker, J. Am. Chem. Soc., 2000, 122, 4092-4097.

8 Y. Zhang, K. Bennett and S. Mukamel, J. Phys. Chem. A, 2018, 122, 6524-6531.

9 G. J. Hedley, A. Ruseckas and I. D. W. Samuel, J. Phys. Chem. A, 2009, 113, 2-4.

10 M. L. Shelby, P. J. Lestrange, N. E. Jackson, K. Haldrup, M. W. Mara, A. B. Stickrath, D. Zhu, H. T. Lemke, M. Chollet, B. M. Hoffman, X. Li and L. X. Chen, J. Am. Chem. Soc., 2016, 138, 8752-8764.

11 M. Maiuri, M. Garavelli and G. Cerullo, J. Am. Chem. Soc., 2020, 142, 3-15.
12 C. N. Fleming, K. A. Maxwell, J. M. DeSimone, T. J. Meyer and J. M. Papanikolas, J. Am. Chem. Soc., 2001, 123, 1033610347.

13 J. K. McCusker, Acc. Chem. Res., 2003, 36, 876-887.

14 M. Iwamura, H. Watanabe, K. Ishii, S. Takeuchi and T. Tahara, J. Am. Chem. Soc., 2011, 133, 7728-7736.

15 M. Chergui, Acc. Chem. Res., 2015, 48, 801-808.

16 M. Iwamura, S. Takeuchi and T. Tahara, Acc. Chem. Res., 2015, 48, 782-791.

17 C. Sousa, C. de Graaf, A. Rudavskyi, R. Broer, J. Tatchen, M. Etinski and C. M. Marian, Chem. - Eur. J., 2013, 19, 17541-17551.

18 W. Yang, B. Ashwood, J. Zhao, W. Ji, D. Escudero, D. Jacquemin and C. E. Crespo-Hernández, J. Phys. Chem. C, 2017, 121, 21184-21198.

19 J. Eng, C. Gourlaouen, E. Gindensperger and C. Daniel, Acc. Chem. Res., 2015, 48, 809-817.

20 G. Capano, U. Rothlisberger, I. Tavernelli and T. J. Penfold, J. Phys. Chem. A, 2015, 119, 7026-7037.

21 K. Li, G. S. Ming Tong, Q. Wan, G. Cheng, W.-Y. Tong, W.H. Ang, W.-L. Kwong and C.-M. Che, Chem. Sci., 2016, 7, 1653-1673.

22 A. D. Kirk, Chem. Rev., 1999, 99, 1607-1640.

23 M. S. Wrighton, Theoretical Inorganic Chemistry II, Berlin, Heidelberg, 1976, pp. 37-104.

24 A. W. Adamson and J. N. Demas, J. Am. Chem. Soc., 1971, 93, 1800-1801.

25 V. Balzani, N. Sabbatini and F. Scandola, Chem. Rev., 1986, 86, 319-337.

26 F. Scandola, M. T. Indelli, C. Chiorboli and C. A. Bignozzi, Photoinduced Electron Transfer II, Berlin, Heidelberg, 1990, pp. 73-149.

27 E. M. Kober, J. L. Marshall, W. J. Dressick, B. P. Sullivan, J. V. Caspar and T. J. Meyer, Inorg. Chem., 1985, 24, 2755-2763.

28 J. F. Endicott, Acc. Chem. Res., 1988, 21, 59-66.

29 H. Rensmo, S. Lunell and H. Siegbahn, J. Photochem. Photobiol. A, 1998, 114, 117 - 124.

30 S. Bonnet, J.-P. Collin and J.-P. Sauvage, Inorg. Chem., 2006, 45, 4024-4034.

31 S. Bonnet, J.-P. Collin and J.-P. Sauvage, Inorg. Chem., 2007, 46, 10520-10533.

32 H. Basch, A. Viste and H. B. Gray, J. Chem. Phys., 1966, 44 10-19.

33 H. B. Gray and C. J. Ballhausen, J. Am. Chem. Soc., 1963 , 85, 260-265.

34 V. Balzani and S. Campagna, Photochemistry and Photophysics of Coordination Compounds I, Springer-Verlag Berlin Heidelberg.

35 T. J. Meyer and J. V. Caspar, Chem. Rev., 1985, 85, 187-218.

36 G. A. Crosby, Acc. Chem. Res., 1975, 8, 231-238.

37 R. J. Watts, J. Chem. Educ., 1983, 60, 834.

38 P. M. Kozlowski, B. D. Garabato, P. Lodowski and M. Jaworska, Dalton Trans., 2016, 45, 4457-4470.

39 K. Kornobis, N. Kumar, P. Lodowski, M. Jaworska, P. Piecuch, 
J. J. Lutz, B. M. Wong and P. M. Kozlowski, J. Comput. Chem., 2013, 34, 987-1004.

40 A. Hauser and C. Reber, in 50 Years of Structure and Bonding - The Anniversary Volume. Structure and Bonding, ed. D. Mingos, Springer, Cham., 2016, ch. Spectroscopy and Chemical Bonding in Transition Metal Complexes.

41 A. Jablonski, Nature., 1933, 131, 839-840.

42 M. Kasha, Discuss. Faraday Soc., 1950, 9, 14-19.

43 L. Paul, T. Moitra, K. Ruud and S. Chakrabarti, J. Phys. Chem. Lett., 2019, 10, 369-374.

44 D. Escudero and W. Thiel, Inorg. Chem., 2014, 53, 1101511019.

45 Q. Zhang, X. Wang, Y. Zhang, L. Wang, J. Li and J. Zhang, J. Phys. Chem. C, 2016, 120, 27523-27532.

46 K. Veys and D. Escudero, J. Phys. Chem. A, 2020, 124, 72287237.

47 C. Feng, S. Li, L. Fu, X. Xiao, Z. Xu, Q. Liao, Y. Wu, J. Yao and $\mathrm{H}$. Fu, J. Phys. Chem. Lett., 0, 0, null.

48 M. Röhrs and D. Escudero, J. Phys. Chem. Lett., 2019, 10, 5798-5804.

49 A. P. Demchenko, V. I. Tomin and P.-T. Chou, Chem. Rev., 2017, 117, 13353-13381.

50 A. Terenin, Acta Physicochim. URSS, 1943, 28, 210-241.

51 G. N. Lewis and M. Kasha, J. Am. Chem. Soc., 1944, 66, 2100-2116.

52 F. Barigelletti, Photochemistry: Volume 38, The Royal Society of Chemistry, 2010, vol. 38, pp. 234-274.

53 K. K.-W. Lo, in Luminescent Transition Metal Complexes as Biological Labels and Probes, ed. V. W. W. Yam, Springer Berlin Heidelberg, Berlin, Heidelberg, 2007, pp. 205-245.

54 S. I. Pascu, P. A. Waghorn, T. D. Conry, H. M. Betts, J. R. Dilworth, G. C. Churchill, T. Pokrovska, M. Christlieb, F. I. Aigbirhio and J. E. Warren, Dalton Trans., 2007, 4988-4997.

55 K. K.-W. Lo, S. P.-Y. Li and K. Y. Zhang, New J. Chem., 2011, 35, 265-287.

56 K. K.-W. Lo, B. T.-N. Chan, H.-W. Liu, K. Y. Zhang, S. P.-Y. Li and T. S.-M. Tang, Chem. Comm., 2013, 49, 4271-4273.

57 Q. Zhao, F. Li and C. Huang, Chem. Soc. Rev., 2010, 39, 3007-3030.

58 Q. Zhao, C. Huang and F. Li, Chem. Soc. Rev., 2011, 40, 2508-2524.

59 K. K.-W. Lo and S. P.-Y. Li, RSC Adv., 2014, 4, 10560-10585.

60 V. Rai-Constapel, S. Salzmann and C. M. Marian, J. Phys. Chem. A, 2011, 115, 8589-8596.

61 M. Etinski, J. Tatchen and C. M. Marian, J. Chem. Phys., 2011, 134, 154105.

62 S. Iuchi and N. Koga, Phys. Chem. Chem. Phys., 2016, 18, 4789-4799.

63 I. Tavernelli, B. F. Curchod and U. Rothlisberger, Chem. Phys., 2011, 391, 101 - 109.

64 S. Mai, P. Marquetand and L. González, J. Chem. Phys., 2014, 140, 204302.

65 M. Pederzoli and J. Pittner, J. Chem. Phys., 2017, 146, 114101.
66 L. Martínez-Fernández, I. Corral, G. Granucci and M. Persico, Chem. Sci., 2014, 5, 1336-1347.

67 M. Pápai, T. J. Penfold and K. B. Mø, J. Phys. Chem. C, 2016, 120, 17234-17241.

68 M. Pápai, G. Vankó, T. Rozgonyi and T. J. Penfold, J. Phys. Chem. Lett., 2016, 7, 2009-2014.

69 S. Mai, P. Marquetand and L. González, Int. J. Quantum Chem., 2015, 115, 1215-1231.

70 S. Mai, P. Marquetand and L. González, J. Phys. Chem. Lett., 2016, 7, 1978-1983.

71 A. Carlos Borin, S. Mai, P. Marquetand and L. González, Phys. Chem. Chem. Phys., 2017, 19, 5888-5894.

72 G. Cui and W. Thiel, J. Chem. Phys., 2014, 141, 124101.

73 S. Mai, M. Richter, P. Marquetand and L. González, Chem. Phys., 2017, 482, 9 - 15.

74 M. Fumanal, E. Gindensperger and C. Daniel, Phys. Chem. Chem. Phys., 2018, 20, 1134-1141.

75 Y. Harabuchi, J. Eng, E. Gindensperger, T. Taketsugu, S. Maeda and C. Daniel, J. Chem. Theory Comput., 2016, 12, 2335-2345.

76 A. J. Atkins and L. González, J. Phys. Chem. Lett., 2017, 8, 3840-3845.

77 S. Gómez, M. Heindl, A. Szabadi and L. González, J. Phys. Chem. A, 2019, 123, 8321-8332.

78 P. A. M. Dirac and R. H. Fowler, Proceedings of the Royal Society of London. Series A, Containing Papers of a Mathematical and Physical Character, 1928, 117, 610-624.

79 K. G. Dyall, J. Chem. Phys., 1994, 100, 2118-2127.

80 W. Pauli, in Zur Quantenmechanik des magnetischen Elektrons, ed. C. P. Enz and K. v. Meyenn, Vieweg+Teubner Verlag, Wiesbaden, 1988, pp. 282-305.

81 F. Neese, J. Chem. Phys., 2005, 122, 034107.

82 G. Schreckenbach and T. Ziegler, Int. J. Quantum Chem., 1997, 61, 899-918.

83 B. A. Hess, C. M. Marian, U. Wahlgren and O. Gropen, Chem. Phys. Lett., 1996, 251, 365 - 371.

84 A. Berning, M. Schweizer, H.-J. Werner, P. J. Knowles and P. Palmieri, Mol. Phys., 2000, 98, 1823-1833.

85 D. G. Fedorov and M. S. Gordona, J. Chem. Phys., 2000, 112, 5611-5623.

86 P. Pokhilko, E. Epifanovsky and A. I. Krylov, J. Chem. Phys., 2019, 151, 034106.

87 M. Blume, R. E. Watson and R. E. Peierls, Proceedings of the Royal Society of London. Series A. Mathematical and Physical Sciences, 1962, 270, 127-143.

88 M. Blume, R. E. Watson and R. E. Peierls, Proceedings of the Royal Society of London. Series A. Mathematical and Physical Sciences, 1963, 271, 565-578.

89 J. S. Cohen, W. R. Wadt and P. J. Hay, J. Chem. Phys., 1979, 71, 2955-2965.

90 C. Ribbing, M. Odelius and J. Kowalewski, Mol. Phys., 1991, 74, 1299-1314.

91 S. Koseki, M. W. Schmidt and M. S. Gordon, J. Phys. Chem., 
1992, 96, 10768-10772.

92 S. Koseki, M. S. Gordon, M. W. Schmidt and N. Matsunaga, J. Phys. Chem., 1995, 99, 12764-12772.

93 S. Koseki, M. W. Schmidt and M. S. Gordon, J. Phys. Chem. A, 1998, 102, 10430-10435.

94 C. Teichteil, M. Pelissier and F. Spiegelmann, Chem. Phys., 1983, 81, $273-282$.

95 L. Seijo and Z. Barandiarán, in The Ab Initio Model Potential Method: A Common Strategy for Effective Core Potential and Embedded Cluster Calculations, pp. 55-152.

96 C. M. Marian and U. Wahlgren, Chem. Phys. Lett., 1996, 251, 357 - 364.

97 S. Yabushita, Z. Zhang and R. M. Pitzer, J. Phys. Chem. A, 1999, 103, 5791-5800.

98 S. R. Langhoff and C. W. Kern, in Molecular Fine Structure, ed. H. F. Schaefer, Springer US, Boston, MA, 1977, pp. 381437.

99 S. G. Chiodo and N. Russo, Chem. Phys. Lett., 2010, 490, 90 $-96$.

100 P. A. Malmqvist, B. Roos and B. Schimmelpfennig, Chem. Phys. Lett., 2002, 357, 230 - 240.

101 F. Rakowitz, M. Casarrubios, L. Seijo and C. M. Marian, J. Chem. Phys., 1998, 108, 7980-7987.

102 E. v. Lenthe, E. J. Baerends and J. G. Snijders, J. Chem. Phys., 1993, 99, 4597-4610.

103 E. van Lenthe, E. J. Baerends and J. G. Snijders, J. Chem. Phys., 1994, 101, 9783-9792.

104 E. van Lenthe, J. G. Snijders and E. J. Baerends, J. Chem. Phys., 1996, 105, 6505-6516.

105 E. van Lenthe, A. Ehlers and E.-J. Baerends, J. Chem. Phys., 1999, 110, 8943-8953.

106 F. Wang and T. Ziegler, J. Chem. Phys., 2005, 123, 154102.

107 K. Mori, T. P. M. Goumans, E. van Lenthe and F. Wang, Phys. Chem. Chem. Phys., 2014, 16, 14523-14530.

108 L. Paul, S. Chakrabarti and K. Ruud, J. Phys. Chem. Lett., 2017, 8, 4893-4897.

109 T. Moitra, M. M. Alam and S. Chakrabarti, Phys. Chem. Chem. Phys., 2018, 20, 23244-23251.

110 S. Mai, H. Gattuso, M. Fumanal, A. Muñoz Losa, A. Monari, C. Daniel and L. González, Phys. Chem. Chem. Phys., 2017, 19, 27240-27250.

111 H. Eschrig and V. D. P. Servedio, J. Comp. Chem., 1999, 20, 23-30.

112 J. Gao, W. Liu, B. Song and C. Liu, J. Chem. Phys., 121, year.

113 J. Gao, W. Zou, W. Liu, Y. Xiao, D. Peng, B. Song and C. Liu, J. Chem. Phys., 2005, 123, 054102.

114 R. Bast, H. Jensen and T. Saue, Int. J. Quantum Chem., 2009, 109, 2091-2112.

115 S. Komorovsky, P. J. Cherry and M. Repisky, J. Chem. Phys., 2019, 151, 184111.

116 S. Villaume, T. Saue and P. Norman, J. Chem. Phys., 2010, 133, 064105.

117 T. Fransson, T. Saue and P. Norman, J. Chem. Theory Comp., 2016, 12, 2324-2334.
118 L. Konecny, M. Repisky, K. Ruud and S. Komorovsky, J. Chem. Phys., 2019, 151, 194112.

119 S. Fraga, K. M. S. Saxena and J. Karwowski, Handbook of atomic data., Elsevier, Amsterdam,The Netherlands, 1976, vol. 5.

120 M. Gernert, L. Balles-Wolf, F. Kerner, U. Müller, A. Schmiedel, M. Holzapfel, C. M. Marian, J. Pflaum, C. Lambert and A. Steffen, J. Am. Chem. Soc., 2020, 142, 8897-8909.

121 G. Baryshnikov, B. Minaev and H. Ågren, Chem. Rev., 2017, 117, 6500-6537.

122 E. N. Harvey, A history of luminescence from the earliest times until 1900, American Philosophical Society, 1957, vol. 44.

123 B. Valeur and M. N. Berberan-Santos, J. Chem. Edu., 2011, 88, 731-738.

124 S. P. McGlynn, T. Azumi, M. Kinoshita et al., 1969.

125 B. Minaev, G. Baryshnikov and H. Agren, Phys. Chem. Chem. Phys., 2014, 16, 1719-1758.

126 A. Messiah and L. I. Schiff, Quantum mechanics, McGraw-Hill College, 1968, vol. 643.

127 S. K. Lower and M. El-Sayed, Chem. Rev., 1966, 66, 199241.

128 Y. Chi and P.-T. Chou, Chem. Soc. Rev., 2007, 36, 1421-1431.

129 P. Norman, K. Ruud and T. Saue, Principles and Practices of Molecular Properties, John Wiley and Sons, Ltd, 1st edn, 2018.

130 Y. Luo, D. Jonsson, P. Norman, K. Ruud, O. Vahtras, B. Minaev, H. Ågren, A. Rizzo and K. Mikkelsen, Int. J. Quantum Chem., 1998, 70, 219-239.

131 E. Jansson, B. Minaev, S. Schrader and H. Ågren, Chem. Phys., 2007, 333, 157-167.

132 B. Minaev, V. Minaeva and H. Ågren, J. Phys. Chem. A, 2009, 113, 726-735.

133 K.-H. Kim, S. Lee, C.-K. Moon, S.-Y. Kim, Y.-S. Park, J.-H. Lee, J. W. Lee, J. Huh, Y. You and J.-J. Kim, Nat. Comm., 2014, 5, $1-8$.

134 S. J. Havriliak and D. R. Yarkony, J. Chem. Phys., 1985, 83, 1168-1172.

135 V. Vallet, L. Maron, C. Teichteil and J.-P. Flament, J. Chem. Phys., 2000, 113, 1391-1402.

136 M. Kleinschmidt, J. Tatchen and C. M. Marian, J. Chem. Phys., 2006, 124, 124101.

137 D. Jacquemin and D. Escudero, Chem. Sci., 2017, 8, 78447850.

138 H. Yu, J. A. Sanchez-Rodriguez, M. Pollum, C. E. CrespoHernández, S. Mai, P. Marquetand, L. González and S. Ullrich, Phys. Chem. Chem. Phys., 2016, 18, 20168-20176.

139 A. Lázaro, C. Cunha, R. Bosque, J. Pina, J. S. Ward, K.-N. Truong, K. Rissanen, J. C. Lima, M. Crespo, J. S. Seixas de Melo and L. Rodríguez, Inorg. Chem., 2020, 59, 8220-8230.

140 F. Geist, A. Jackel and R. F. Winter, Inorg. Chem., 2015, 54, 10946-10957.

141 F. Geist, A. Jackel and R. F. Winter, Dalton Trans., 2015, 44, 3974-3987. 
142 Y. You and S. Y. Park, Dalton Trans., 2009, 1267-1282.

143 A. Haque, L. Xu, R. A. Al-Balushi, M. K. Al-Suti, R. Ilmi, Z. Guo, M. S. Khan, W.-Y. Wong and P. R. Raithby, Chem. Soc. Rev., 2019, 48, 5547-5563.

144 C. Song, J. Tang, J. Li, Z. Wang, P. Li and H. Zhang, Inorg. Chem., 2018, 57, 12174-12186.

145 S. Y.-L. Leung, E. S.-H. Lam, W. H. Lam, K. M.-C. Wong, W.-T. Wong and V. W.-W. Yam, Chem. - Eur. J., 2013, 19, 10360-10369.

146 G. Li, X. Zhao, T. Fleetham, Q. Chen, F. Zhan, J. Zheng, Y.-F. Yang, W. Lou, Y. Yang, K. Fang, Z. Shao, Q. Zhang and Y. She, Chem. Mater., 2020, 32, 537-548.

147 T. Fleetham, G. Li, L. Wen and J. Li, Adv. Mater., 2014, 26, 7116-7121.

148 C. Lee, R. Zaen, K.-M. Park, K. H. Lee, J. Y. Lee and Y. Kang, Organometallics, 2018, 37, 4639-4647.

149 M. Krause, D. Kourkoulos, D. González-Abradelo, K. Meerholz, C. A. Strassert and A. Klein, Eur. J. Inorg. Chem., 2017, 2017, 5215-5223.

150 P. J. G. Saris and M. E. Thompson, Org. Lett., 2016, 18, 3960-3963.

151 J. Soellner and T. Strassner, Organometallics, 2018, 37, 1821-1824.

152 J. D. Bullock, A. Salehi, C. J. Zeman, K. A. Abboud, F. So and K. S. Schanze, ACS App. Mater. Inter., 2017, 9, 41111-41114.

153 G. A. Worth, M. H. Beck, A. Jäckle and H.-D. Meyer, The MCTDH package, 2011, http://mctdh.uni-hd.de.

154 H. Wang and M. Thoss, J. Chem. Phys., 2003, 119, 12891299.

155 H. Meyer, F. Gatti and G. Worth, Weinheim: VCH. Tannor, DJ (2007)., 2008.

156 F. Plasser, S. Gomez, M. F. S. J. Menger, S. Mai and L. Gonzalez, Phys. Chem. Chem. Phys., 2019, 21, 57-69.

157 J. C. Tully and R. K. Preston, J. Chem. Phys., 1971, 55, 562572.

158 T. J. Martinez, M. Ben-Nun and R. Levine, J. Phys. Chem., 1996, 100, 7884-7895.

159 E. J. Heller, J. Chem. Phys., 1975, 62, 1544-1555.

160 T. J. Penfold, E. Gindensperger, C. Daniel and C. M. Marian, Chem. Rev., 2018, 118, 6975-7025.

161 R. Borrelli and A. Peluso, J. Chem. Phys., 2003, 119, 84378448.

162 J. R. Reimers, J. Chem. Phys., 2001, 115, 9103-9109.

163 M. El-Sayed, J. Chem. Phys., 1963, 38, 2834-2838.

164 R. Englman and J. Jortner, Mol. Phys., 1970, 18, 145-164.

165 A. Toniolo and M. Persico, J. Comput. Chem., 2001, 22, 968975.

166 J. Tatchen, N. Gilka and C. M. Marian, Phys. Chem. Chem. Phys., 2007, 9, 5209-5221.

167 M. Dierksen and S. Grimme, J. Chem. Phys., 2005, 122, 244101

168 F. Santoro, A. Lami, R. Improta, J. Bloino and V. Barone, J. Chem. Phys., 2008, 128, 224311.

169 K. Schmidt, S. Brovelli, V. Coropceanu, D. Beljonne,
J. Cornil, C. Bazzini, T. Caronna, R. Tubino, F. Meinardi, Z. Shuai et al., J. Phys. Chem. A, 2007, 111, 10490-10499.

170 D. J. Tannor, Introduction to quantum mechanics: a timedependent perspective, University Science Books, 2007.

171 R. Kubo, Phys. Rev., 1952, 86, 929-937.

172 M. Lax, J. Chem. Phys., 1952, 20, 1752-1760.

173 R. Kubo and Y. Toyozawa, Prog. Theor. Phys., 1955, 13, 160182.

174 P. Karak and S. Chakrabarti, Submitted.

175 R. Borrelli and A. Peluso, WIREs Comput. Mol. Sc., 2013, 3, 542-559.

176 C. M. Marian, WIREs Comput. Mol. Sci., 2012, 2, 187-203.

177 R. Borrelli and A. Peluso, J. Chem. Theory Comput., 2015, 11, 415-422.

178 A. Velardo, R. Borrelli, A. Peluso and A. Capobianco, J. Phys. Chem. C, 2016, 120, 24605-24614.

179 A. Landi, R. Borrelli, A. Capobianco, A. Velardo and A. Peluso, J. Phys. Chem. C, 2018, 122, 25849-25857.

180 R. Borrelli and A. Peluso, Phys. Chem. Chem. Phys., 2011, 13, 4420-4426.

181 R. Borrelli, M. Thoss, H. Wang and W. Domcke, Mol. Phys., 2012, 110, 751-763.

182 N. Lüdtke, J. Föller and C. M. Marian, Phys. Chem. Chem. Phys., 2020, -.

183 I. Lyskov and C. M. Marian, J. Phys. Chem. C, 2017, 121, 21145-21153.

184 J. Föller, M. Kleinschmidt and C. M. Marian, Inorg. Chem., 2016, 55, 7508-7516.

185 A. Heil, K. Gollnisch, M. Kleinschmidt and C. M. Marian, Mol. Phys., 2016, 114, 407-422.

186 M. Kleinschmidt, C. van Wüllen and C. M. Marian, J. Chem. Phys., 2015, 142, 094301.

187 E. Yu-Tzu Li, T.-Y. Jiang, Y. Chi and P.-T. Chou, Phys. Chem. Chem. Phys., 2014, 16, 26184-26192.

188 K. T. Chan, G. S. M. Tong, W.-P. To, C. Yang, L. Du, D. L. Phillips and C.-M. Che, Chem. Sci., 2017, 8, 2352-2364.

189 M. Z. Shafikov, D. N. Kozhevnikov, M. Bodensteiner, F. Brandl and R. Czerwieniec, Inorg. Chem., 2016, 55, 74577466.

190 Y. Yu, S. Mallick, M. Wang and K. Borjesson, 2020.

191 D. Polak, R. Jayaprakash, T. P. Lyons, L. A. MartínezMartínez, A. Leventis, K. J. Fallon, H. Coulthard, D. G. Bossanyi, K. Georgiou, A. J. Petty, II, J. Anthony, H. Bronstein, J. Yuen-Zhou, A. I. Tartakovskii, J. Clark and A. J. Musser, Chem. Sci., 2020, 11, 343-354.

192 H. Li, A. Kamasah, S. Matsika and A. G. Suits, Nat. Chem., 2019, 11, 123-138.

193 R. Monni, G. Capano, G. Auböck, H. B. Gray, A. Vlček, I. Tavernelli and M. Chergui, Proc. Natl. Acad. Sci., 2018, 115, E6396-E6403.

194 L. Mewes, R. A. Ingle, S. Megow, H. Böhnke, E. Baranoff, F. Temps and M. Chergui, Inorg. Chem., 2020, 59, 1464314653. 
195 C. Imberti, P. Zhang, H. Huang and P. J. Sadler, Angew. Chem. Int. Ed., 2020, 59, 61-73.

196 S. Mai and L. Gonzalez, Angew. Chem. Int. Ed., 2020, 59, 16832-16846.

197 A. J. S. Valentine and X. Li, J. Chem. Phys., 2019, 151, 084107.

198 P. Pokhilko and A. I. Krylov, J. Phys. Chem. Lett., 2019, 10, 4857-4862.

199 R. Borrelli and M. Gelin, Sci. Rep., 2017, 7, 9127.

200 I. Kim, S. O. Jeon, D. Jeong, H. Choi, W.-J. Son, D. Kim, Y. M. Rhee and H. S. Lee, J. Chem. Theo. Comput., 2020, 16, 621-632.

201 F. Talotta, S. Morisset, N. Rougeau, D. Lauvergnat and F. Agostini, J. Chem. Theo. Comput., 2020, 16, 4833-4848.

202 P. O. Dral, M. Barbatti and W. Thiel, J. Phys. Chem. Lett., 2018, 9, 5660-5663.

203 J. Zhao, S. Ji, W. Wu, W. Wu, H. Guo, J. Sun, H. Sun, Y. Liu, Q. Li and L. Huang, RSC Adv., 2012, 2, 1712-1728.

204 K. K.-W. Lo, S. P.-Y. Li and K. Y. Zhang, New J. Chem., 2011, 35, 265-287.

205 A. Ahmad Warra, J. Chem. Pharma. Res., 2011, vol 3, pp 951-958.

206 C. Ulbricht, B. Beyer, C. Friebe, A. Winter and U. Schubert, Adv. Mater., 2009, 21, 4418 - 4441.

207 C. Humphreys, MRS Bulletin, 2008, 33,.

208 K. Kamtekar, A. Monkman and M. Bryce, Adv. Mater., 2010, 22, 572-82.

209 I. M. Dixon, J.-P. Collin, J.-P. Sauvage, L. Flamigni, S. Encinas and F. Barigelletti, Chem. Soc. Rev., 2000, 29, 385-391.

210 V. Marin, E. Holder, R. Hoogenboom and U. S. Schubert, Chem. Soc. Rev., 2007, 36, 618-635.

211 J. D. Slinker, J. Rivnay, J. S. Moskowitz, J. B. Parker, S. Bernhard, H. D. Abruña and G. G. Malliaras, J. Mater. Chem., 2007, 17, 2976-2988.

212 M. Thompson, MRS Bulletin, 2007, 32, 694-701.

213 W.-Y. W. Q. W. D. M. L. W. Z. L. T. B. M. Guijiang Zhou, Cheuk-Lam Ho and A. Beeby, Adv. Funct. Mater, 2008, 18, 499.

214 K. A. Knights, S. G. Stevenson, C. P. Shipley, S.-C. Lo, S. Olsen, R. E. Harding, S. Gambino, P. L. Burn and I. D. W. Samuel, J. Mater. Chem., 2008, 18, 2121-2130.

215 S. Vickers, H. Barcena, K. A. Knights, R. Thomas, J.-C. Ribierre, S. Gambino, I. Samuel, P. Burn and G. Fragneto, App. Phys. Lett., 2010, 96, 263302.

216 A. W. Freeman, S. C. Koene, P. R. L. Malenfant, M. E. Thompson and J. M. J. Fréchet, J. Am. Chem. Soc., 2000, 49, 122.

217 Org. Electron., 2006, 7, 85-98.

218 Chem. Sci., 2015, 6, 4623-4635.

219 P. Pander, R. Bulmer, R. Martinscroft, S. Thompson, F. W. Lewis, T. J. Penfold, F. B. Dias and V. N. Kozhevnikov, Inorg. Chem., 2018, 57, 3825-3832.

220 G. Cheng, S. C. F. Kui, W.-H. Ang, M.-Y. Ko, P.-K. Chow, C.-L. Kwong, C.-C. Kwok, C. Ma, X. Guan, K.-H. Low, S.-J. Su and C.-M. Che, Chem. Sci., 2014, 5, 4819-4830.
221 K. Li, G. Cheng, C. Ma, X. Guan, W.-M. Kwok, Y. Chen, W. Lu and C.-M. Che, Chem. Sci., 2013, 4, 2630-2644.

222 J. C. deMello, J. J. M. Halls, S. C. Graham, N. Tessler and R. H. Friend, Phys. Rev. Lett., 2000, 85, 421-424.

223 Q. Pei, Y. Yang, G. Yu, C. Zhang and A. J. Heeger, J. Am. Chem. Soc., 1996, 118, 3922-3929.

224 R. D. Costa, E. Ortí, H. J. Bolink, F. Monti, G. Accorsi and N. Armaroli, Angew. Chem. (Int. ed. Eng.), 2012, 51, 81788211.

225 K. M. Maness, R. H. Terrill, T. J. Meyer, R. W. Murray and R. M. Wightman, J. Am. Chem. Soc., 1996, 118, 1060910616.

226 J. D. Slinker, A. A. Gorodetsky, M. S. Lowry, J. Wang, S. Parker, R. Rohl, S. Bernhard and G. G. Malliaras, J. Am. Chem. Soc., 2004, 126, 2763-2767.

227 D.-L. Ma, H.-Z. He, K.-H. Leung, D. S.-H. Chan and C.-H. Leung, Angew. Chem. (Int. ed. Eng.), 2013, 52, 7666-7682.

228 H.-K. Liu and P. J. Sadler, Acc. Chem. Res., 2011, 44, 349359.

229 M. Yu, Q. Zhao, L. Shi, F. Li, Z. Zhou, H. Yang, T. Yi and C. Huang, Chem. Comm., 2008, 2115-2117.

230 A. E. Friedman, J. C. Chambron, J. P. Sauvage, N. J. Turro and J. K. Barton, J. Am. Chem. Soc., 1990, 112, 4960-4962.

231 C. Turro, S. H. Bossmann, Y. Jenkins, J. K. Barton and N. J. Turro, J. Am. Chem. Soc., 1995, 117, 9026-9032.

232 K. Suhling, P. M. W. French and D. Phillips, Photochem. Photobiol. Sci., 2005, 4, 13-22.

233 F. R. Svensson, M. Abrahamsson, N. StrÃümberg, A. G. Ewing and P. Lincoln, J. Phys. Chem. Lett., 2011, 2, 397-401.

234 L. Huynh, Z. Wang, J. Yang, V. Stoeva, A. Lough, I. Manners and M. A. Winnik, Chem. Mater., 2005, 17, 4765-4773.

235 K. Aidas, C. Angeli, K. L. Bak, V. Bakken, R. Bast, L. Boman, O. Christiansen, R. Cimiraglia, S. Coriani, P. Dahle, E. K. Dalskov, U. Ekström, T. Enevoldsen, J. J. Eriksen, P. Ettenhuber, B. Fernández, L. Ferrighi, H. Fliegl, L. Frediani, K. Hald, A. Halkier, C. Hättig, H. Heiberg, T. Helgaker, A. C. Hennum, H. Hettema, E. Hjertenaes, S. Høst, I.-M. Høyvik, M. F. Iozzi, B. Jansík, H. J. A. Jensen, D. Jonsson, P. Jørgensen, J. Kauczor, S. Kirpekar, T. Kjaergaard, W. Klopper, S. Knecht, R. Kobayashi, H. Koch, J. Kongsted, A. Krapp, K. Kristensen, A. Ligabue, O. B. Lutnaes, J. I. Melo, K. V. Mikkelsen, R. H. Myhre, C. Neiss, C. B. Nielsen, P. Norman, J. Olsen, J. M. H. Olsen, A. Osted, M. J. Packer, F. Pawlowski, T. B. Pedersen, P. F. Provasi, S. Reine, Z. Rinkevicius, T. A. Ruden, K. Ruud, V. V. Rybkin, P. Salek, C. C. M. Samson, A. S. de Merás, T. Saue, S. P. A. Sauer, B. Schimmelpfennig, K. Sneskov, A. H. Steindal, K. O. Sylvester-Hvid, P. R. Taylor, A. M. Teale, E. I. Tellgren, D. P. Tew, A. J. Thorvaldsen, L. Thøgersen, O. Vahtras, M. A. Watson, D. J. D. Wilson, M. Ziolkowski and H. Ågren, WIREs Comp. Mol. Sc., 2014, 4, 269-284.

236 Y. Shao, Z. Gan, E. Epifanovsky, A. T. Gilbert, M. Wormit, J. Kussmann, A. W. Lange, A. Behn, J. Deng, X. Feng, D. Ghosh, M. Goldey, P. R. Horn, L. D. Jacobson, I. Kali- 
man, R. Z. Khaliullin, T. Kuś, A. Landau, J. Liu, E. I. Proynov, Y. M. Rhee, R. M. Richard, M. A. Rohrdanz, R. P. Steele, E. J. Sundstrom, H. L. W. III, P. M. Zimmerman, D. Zuev, B. Albrecht, E. Alguire, B. Austin, G. J. O. Beran, Y. A. Bernard, E. Berquist, K. Brandhorst, K. B. Bravaya, S. T. Brown, D. Casanova, C.-M. Chang, Y. Chen, S. H. Chien, K. D. Closser, D. L. Crittenden, M. Diedenhofen, R. A. D. Jr., H. Do, A. D. Dutoi, R. G. Edgar, S. Fatehi, L. Fusti-Molnar, A. Ghysels, A. Golubeva-Zadorozhnaya, J. Gomes, M. W. HansonHeine, P. H. Harbach, A. W. Hauser, E. G. Hohenstein, Z. C. Holden, T.-C. Jagau, H. Ji, B. Kaduk, K. Khistyaev, J. Kim, J. Kim, R. A. King, P. Klunzinger, D. Kosenkov, T. Kowalczyk, C. M. Krauter, K. U. Lao, A. D. Laurent, K. V. Lawler, S. V. Levchenko, C. Y. Lin, F. Liu, E. Livshits, R. C. Lochan, A. Luenser, P. Manohar, S. F. Manzer, S.-P. Mao, N. Mardirossian, A. V. Marenich, S. A. Maurer, N. J. Mayhall, E. Neuscamman, C. M. Oana, R. Olivares-Amaya, D. P. O’Neill, J. A. Parkhill, T. M. Perrine, R. Peverati, A. Prociuk, D. R. Rehn, E. Rosta, N. J. Russ, S. M. Sharada, S. Sharma, D. W. Small, A. Sodt, T. Stein, D. Stück, Y.-C. Su, A. J. Thom, T. Tsuchimochi, V. Vanovschi, L. Vogt, O. Vydrov, T. Wang, M. A. Watson, J. Wenzel, A. White, C. F. Williams, J. Yang, S. Yeganeh, S. R. Yost, Z.-Q. You, I. Y. Zhang, X. Zhang, Y. Zhao, B. R. Brooks, G. K. Chan, D. M. Chipman, C. J. Cramer, W. A. G. III, M. S. Gordon, W. J. Hehre, A. Klamt, H. F. S. III, M. W. Schmidt, C. D. Sherrill, D. G. Truhlar, A. Warshel, X. Xu, A. Aspuru-Guzik, R. Baer, A. T. Bell, N. A. Besley, J.-D. Chai, A. Dreuw, B. D. Dunietz, T. R. Furlani, S. R. Gwaltney, C.-P. Hsu, Y. Jung, J. Kong, D. S. Lambrecht, W. Liang, C. Ochsenfeld, V. A. Rassolov, L. V. Slipchenko, J. E. Subotnik, T. V. Voorhis, J. M. Herbert, A. I. Krylov, P. M. Gill and M. HeadGordon, Mol. Phys., 2015, 113, 184-215.

237 M. J. Frisch, G. W. Trucks, H. B. Schlegel, G. E. Scuseria, M. A. Robb, J. R. Cheeseman, G. Scalmani, V. Barone, G. A.
Petersson, H. Nakatsuji, X. Li, M. Caricato, A. V. Marenich, J. Bloino, B. G. Janesko, R. Gomperts, B. Mennucci, H. P. Hratchian, J. V. Ortiz, A. F. Izmaylov, J. L. Sonnenberg, D. Williams-Young, F. Ding, F. Lipparini, F. Egidi, J. Goings, B. Peng, A. Petrone, T. Henderson, D. Ranasinghe, V. G. Zakrzewski, J. Gao, N. Rega, G. Zheng, W. Liang, M. Hada, M. Ehara, K. Toyota, R. Fukuda, J. Hasegawa, M. Ishida, T. Nakajima, Y. Honda, O. Kitao, H. Nakai, T. Vreven, K. Throssell, J. A. Montgomery, Jr., J. E. Peralta, F. Ogliaro, M. J. Bearpark, J. J. Heyd, E. N. Brothers, K. N. Kudin, V. N. Staroverov, T. A. Keith, R. Kobayashi, J. Normand, K. Raghavachari, A. P. Rendell, J. C. Burant, S. S. Iyengar, J. Tomasi, M. Cossi, J. M. Millam, M. Klene, C. Adamo, R. Cammi, J. W. Ochterski, R. L. Martin, K. Morokuma, O. Farkas, J. B. Foresman and D. J. Fox, Gaussian16 Revision C.01, 2016, Gaussian Inc. Wallingford CT.

238 C. M. Marian, A. Heil and M. Kleinschmidt, WIREs Comput. Mol. Sc., 2019, 9, e1394.

239 G. te Velde, F. M. Bickelhaupt, E. J. Baerends, C. Fonseca Guerra, S. J. A. van Gisbergen, J. G. Snijders and T. Ziegler, J. Comput. Chem., 2001, 22, 931-967.

240 M. Repisky, S. Komorovsky, M. Kadek, L. Konecny, U. Ekström, E. Malkin, M. Kaupp, K. Ruud, O. L. Malkina and V. G. Malkin, J. Chem. Phys., 2020, 152, 184101.

241 D. A. Matthews, L. Cheng, M. E. Harding, F. Lipparini, S. Stopkowicz, T.-C. Jagau, P. G. Szalay, J. Gauss and J. F. Stanton, J. Chem. Phys., 2020, 152, 214108.

242 F. Santoro, R. Improta, A. Lami, J. Bloino and V. Barone, J. Chem. Phys., 2007, 126, 084509.

243 S. Mai, M. Richter, M. Heindl, M. Menger, A. Atkins, M. Ruckenbauer, F. Plasser, L. Ibele, S. Kropf, M. Oppel, P. Marquetand and L. González, SHARC2.1: Surface Hopping Including Arbitrary Couplings - Program Package for Non-Adiabatic Dynamics, 2019, sharc-md.org. 